\title{
Analysis of blinkers and EUV brightenings in the quiet Sun observed with CDS
}

\author{
A. Brković ${ }^{1}$, S. K. Solanki², and I. Rüedi ${ }^{3}$ \\ 1 Institute of Astronomy, ETH-Zentrum, 8092 Zürich, Switzerland \\ 2 Max-Planck-Institut für Aeronomie, Max-Planck-Str. 2, 37191 Katlenburg-Lindau, Germany \\ 3 Physikalisch-Meteorologisches Observatorium Davos, World Radiation Center, 7260 Davos Dorf, Switzerland
}

Received 28 February 2001 / Accepted 23 April 2001

\begin{abstract}
Movies of quiet Sun regions at disc centre obtained with the Coronal Diagnostic Spectrometer (CDS) onboard the SOHO spacecraft are used to study the properties of transient brightenings seen in the extreme ultraviolet (EUV), so-called blinkers, at three different temperatures sampled simultaneously in the chromospheric He I $584.3 \AA\left(2 \times 10^{4} \mathrm{~K}\right)$, the transition region O V $629.7 \AA\left(2.5 \times 10^{5} \mathrm{~K}\right)$ and coronal $\mathrm{Mg} \mathrm{IX} 368.1 \AA\left(10^{6} \mathrm{~K}\right)$ lines. Blinkers, here defined somewhat differently than in previous studies, were clearly detected in the $\mathrm{O} V$ and He I lines. Brightenings of the Mg IX line were also seen. A thorough analysis of blinker properties is carried out and their detailed properties are determined. Blinkers are found to be present in both bright (network) and dark (intranetwork) regions, but their number density is larger in the brighter areas (in $\mathrm{O} \mathrm{V}$ ) although the rest of their properties appear to be unaffected. The average sizes of brightenings range from $2.8 \mathrm{Mm}^{2}$ in $\mathrm{Mg}$ IX, $12.4 \mathrm{Mm}^{2}$ in He I to $23.5 \mathrm{Mm}^{2}$ in $\mathrm{O} \mathrm{V}$. The durations of blinkers are in the range $3-110 \mathrm{~min}$, with the average durations being $23 \mathrm{~min}$ in $\mathrm{He} \mathrm{I}$, about $16 \mathrm{~min}$ in $\mathrm{O} \mathrm{V}$ and $12 \mathrm{~min}$ in $\mathrm{Mg}$ IX. The frequency distributions of ratio of peak to background intensity, excess energy and size follow power laws with exponents $<-5$ for the intensity ratio, and between -1 and -3 for the other two parameters. The correlation coefficients between pairs of ratio, energy and size are at least 0.5 , while other pairs of parameters describing the blinkers appear to be uncorrelated. The best correlation is between size and energy. The blinker durations exhibit a distribution whose form is compatible with a log-normal function. Finally, blinkers in the 3 lines (i.e. 3 temperature regimes) are poorly correlated; with the correlation coefficient being always less than 0.4. This suggests that to a large extent the transition region reacts independently of the corona and chromosphere to energy deposition, so that these parts of the atmosphere are at least partly decoupled from each other. This agrees with the expectations from models having separate transition-region loops, but contradicts the classical picture of the transition region, as being heated dominantly by energy conduction from the corona.
\end{abstract}

Key words. Sun: chromosphere - Sun: corona - Sun: transition region - Sun: UV radiation

\section{Introduction}

Harrison (1997) first used the term blinker for extreme ultraviolet (EUV) brightenings in the quiet Sun showing an enhancement of a factor 2-3 in the flux of transition region lines. These blinkers have durations in the range of 1 to $30 \mathrm{~min}$, with an average of $13 \mathrm{~min}$. Their thermal energy content is reported to be on the order of $10^{25} \mathrm{erg}$ or $10^{-6}$ times that of a 'standard' flare. Harrison et al. (1999) studied the properties of blinkers in greater detail and presented a blinker model. They found that the most significant brightenings are observed in the lines of O III, $\mathrm{O} \mathrm{IV}$ and $\mathrm{O} \mathrm{V}$ formed at transition region temperatures. Lower (He I) or higher ( $\mathrm{Mg} \mathrm{IX}$ and $\mathrm{Mg} \mathrm{X}$ ) temperature lines did not reveal as significant an increase in brightness.

Send offprint requests to: A. Brković,

e-mail: brkovic@astro.phys.ethz.ch
According to the Harrison et al. report a typical blinker lasts about $1000 \mathrm{~s}$, but due to the fact that the distribution of blinker lifetimes has a long-duration tail, the average duration is $40 \mathrm{~min}$. The number of blinkers occurring on the Sun at any point in time was estimated to be approximately 3000 with a birth rate of $1.24 \mathrm{~s}^{-1}$. The same report pointed out that blinkers are mainly caused by increases in density or filling factor and are not due to a temperature increase of the plasma.

Berghmans et al. (1998) studied quiet-Sun EUV transient brightenings by applying a Fourier analysis to data obtained with the Extreme-Ultraviolet Imaging Telescope (EIT) onboard SOHO, and reported on brightenings in He II $304 \AA$ (formed in the cooler parts of the transition region) lasting from 2 to $60 \mathrm{~min}$, while brightenings in the low corona observed with the Fe XII $195 \AA$ line range from 2 to $30 \mathrm{~min}$. They propose that the transition region 
brightenings seen in He II are in fact blinkers, as defined by Harrison (1997).

Other forms of transient activity have been recorded in the quiet Sun as well. These differ from blinkers in the temperature regime in which they are studied or in the techniques used to identify them. For example, Krucker et al. (1997) and Krucker \& Benz (1998) have studied the statistics of flare-like events, called network flares, using the Soft X-ray Telescope (SXT) on Yohkoh, EIT on SOHO and the Very Large Array (VLA). These observations refer to gas at coronal temperatures, i.e. above a million K. If network flares are related to blinkers their signatures would have been detected in the Mg IX and Mg X lines used in blinker studies (Harrison et al. 1999). Blinkers are probably also unrelated to explosive events, identified by Brueckner \& Bartoe (1983) using the HRTS instrument. These manifest themselves as localized broadenings of transition region lines, and are not necessarily associated with a brightening (Dere et al. 1984). Innes et al. (1997), using SUMER on SOHO, showed that explosive events correspond closely to the signature expected for magnetic reconnection. Finally, Brković et al. (2000) investigated events exhibiting a high variability in brightness. So far no firm relationship between the blinkers, the explosive events and the network flares has been established. One of the questions that we intend to address is the relation between transient brightenings in the chromosphere, transition region and corona by comparing simultaneous observations at three different temperatures between $20000 \mathrm{~K}$ and $10^{6} \mathrm{~K}$.

Transient brightenings are thought to be the signature of heating episodes, or rather of the cooling after the heating episodes (e.g. Harrison et al. 1999). Studying them thus could help to determine just how important such heating episodes are for maintaining the thermal structure of the corona and transition region. For example, there has been considerable interest in the distribution of the energy in transient brightenings (e.g., Hudson 1991; Crosby et al. 1993; Krucker \& Benz 1998; Parnell \& Jupp 2000), since it gives us an indication whether there is sufficient energy in such events to heat the corona or not.

Also, a comparison between brightenings at different temperatures could help to distinguish between models of the solar transition region. In the standard plane-parallel model of the solar atmosphere and its extension by Gabriel (1976) to include the expansion of the magnetic field with height, the whole transition region is connected directly to the corona and responds mainly to heating in the corona through the downward conduction of energy. In the alternative model of Dowdy et al. (1986), on the other hand, a significant part of the radiation at transition region temperatures comes from cool loops that do not harbour any gas at coronal temperatures. In the former case we expect a high correlation between brightenings at coronal and transition region temperatures, whereas in the latter case only a subset of the brightenings are expected to be related, namely those associated with transition-region gas located along field lines that reach into the corona.
A comparison between transient brightenings in chromospheric and coronal layers should thus help to reveal how strongly these are coupled.

In this paper we present the results of a study of the blinker activity observed in the quiet Sun using movies, obtained with the Coronal Diagnostic Spectrometer (CDS). We also compare blinkers to other transient events in the quiet Sun. A preliminary version of some of the results was presented by Brković et al. (1999).

\section{Observations}

The analysed observations have been carried out using the Normal Incidence Spectrometer (NIS) of CDS (Harrison et al. 1995) in its movie mode, i.e. with the $90^{\prime \prime} \times 240^{\prime \prime}$ slit. In this mode a filtergram covering a part of the solar surface corresponding to the slit size is produced at each wavelength. Filtergrams in 3 strong and sufficiently isolated spectral lines, He I $584.3 \AA\left(2 \times 10^{4} \mathrm{~K}\right), \mathrm{O} \mathrm{V}$ $629.7 \AA\left(2.5 \times 10^{5} \mathrm{~K}\right)$ and $\mathrm{Mg} \mathrm{IX} 368.1 \AA\left(10^{6} \mathrm{~K}\right)$, were recorded simultaneously. Spectral information within each spectral line is lost in this mode. The images were obtained at a cadence of $31 \mathrm{~s}$ for a duration of 4 hours each on 3rd December 1996 and on 6th December 1996. The actual exposure time was $25 \mathrm{~s}$ and the overhead per frame amounted to 6 seconds (the overhead is mainly required to read out the CCD and to prepare it for the next exposure). The targets were quiet regions at the centre of the solar disc, observed at a time of low solar activity.

After carrying out the standard reduction procedure - i.e., correcting for bias of the detector, calibration, etc. - we used cross-correlations and pointing information provided by the instrument housekeeping data to compensate for solar rotation and changes in the telescope pointing. Therefore, in the reduced time series, each pixel follows the same point on the solar surface during the whole time series. The field of view is reduced by this procedure, however. Therefore, the final analysed field of view is $67^{\prime \prime} \times 217^{\prime \prime}(40 \times 129$ pixels $)$, with the pixel size being $1.68^{\prime \prime} \times 1.68^{\prime \prime}$. We took particular care to interpolate over pixels with missing data and pixels contaminated by cosmic rays. Cosmic rays could easily mimic a small blinker event and therefore had to be rejected. This was performed using the CDS software procedure CDS_CLEAN. The procedure searches for isolated pixels in a given exposure which are very bright compared to neighbouring exposures and more than $3 \sigma$ brighter than their neighbours. As we show later in this paper (Fig. 8) the number of blinkers decreases very rapidly with decreasing duration below $10 \mathrm{~min}$, so that we do not expect that we reject many real blinkers through this procedure. Finally, the data were corrected, to first order, for the slow evolution of the geometrical structure of solar features by removing the long-term linear trend. This was done separately for each pixel and spectral line. The signal to noise ratios averaged over space and time are 19.4 and 21.0 for He I, 13.3 and 14.6 for $\mathrm{O} \mathrm{V}$ and 7.8 and 7.7 for $\mathrm{Mg}$ IX for the two data sets, respectively. The data are the same as analysed 


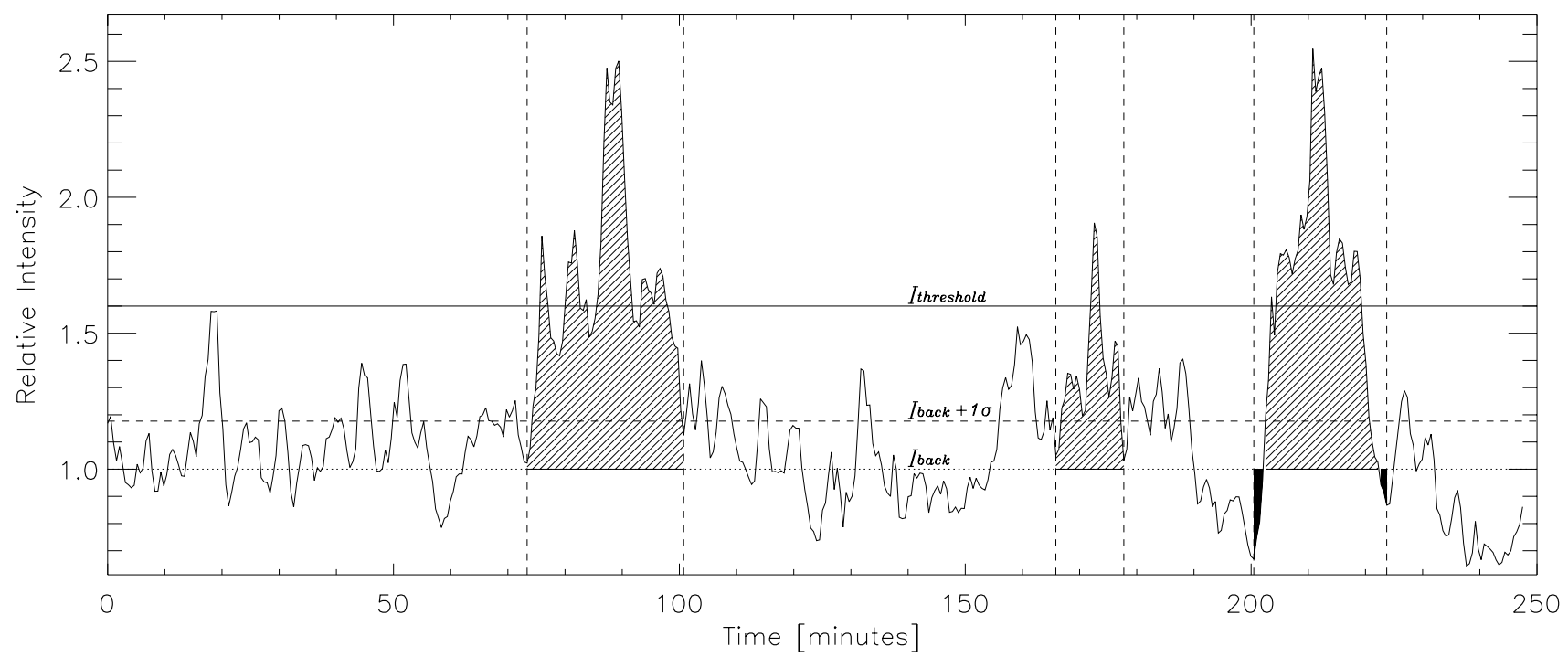

Fig. 1. Sample light curve in O V displaying 3 events classified as blinkers. The vertical axis shows the intensity relative to the averaged background intensity (horizontal dotted line). The horizontal lines signify the following: Dotted: the value of the background intensity after iterative removal of the blinker contributions; dashed: the value of the background increased by $1 \sigma$, i.e. by its standard deviation; solid: the threshold intensity used to identify blinkers. The vertical dashed lines represent the start and end times of the 3 blinkers. The areas of the shaded regions are proportional to the energies radiated by the blinkers (but see the text).

by Brković et al. (2000) and more details can be found there.

\section{Blinker identification}

\subsection{Blinker identification}

First we need to identify the blinkers. For this we roughly follow the criterion used by Harrison (1997) that blinkers are brightenings that exceed a certain threshold. However, in order to make the identification and the determination of the parameters of blinkers automatic and objective we have worked out an elaborate iterative scheme which deviates somewhat from his approach. Note, however, that some amount of arbitrariness cannot be avoided, and other, equally reasonable criteria could lead to somewhat different properties of blinkers.

Figure 1 shows the light curve of a single pixel which we use to illustrate the 4 steps of our method of identifying blinkers. Step 1: for each pixel we first determine the time steps at which the intensity is above a given threshold that is chosen to be some factor times the time-averaged intensity of that pixel (solid horizontal line in Fig. 1). This threshold is different for each spectral line and the reasons for the adopted choices are described below. Step 2: starting from the local maximum lying above the threshold we follow the intensity both backward and forward in time, until local minima are found which lie below the timeaveraged intensity of the pixel in question (termed the background intensity). These points are designated as the preliminary start and end times of a brightening event. Step 3: if one or more brightness events are identified in a particular pixel a new background intensity is calculated by averaging the intensities of only those time steps not harbouring such events. Steps 1-3 were then repeated with the new (lower) background intensity (which also implies a lowering of the threshold intensity, since the ratio to the background is kept fixed) until no new event is identified. This was the case after less than 5 iterations for all spatial pixels in our data set. As an illustration in Fig. 1 a threshold of 1.6 leads to the identification of 3 blinkers with different durations. From now on all intensities are given relative to this final background intensity. Finally, in Step 4 we determine the standard deviation $(\sigma)$ of the intensity at the time-steps not harbouring brightness events (i.e. of the background intensity) and we repeat step 2, but this time looking for local minima below the final background intensity plus the $1 \sigma$ standard deviation above this value (horizontal dashed line in Fig. 1). This further step was introduced since in a number of cases brightness events with extremely long lifetimes were found, which only had a single relatively short-lived peak lying above the threshold, however. Since in most such cases the intensity just fails to drop below the background relatively close to the peak we decided that these long lifetimes are often an artifact of noise or intrinsic, weak background variability and introduced step 4 . The second of the three brightenings in Fig. 1 is an example of an event whose lifetime has been shortened by this step.

Since some brightness events started before the beginning or ended after the end of our observations their starting and ending times cannot be determined and consequently they are not retained for further analysis.

\subsection{Spatial grouping}

In many cases not just an isolated pixel exhibits a brightness event, but rather a group of pixels appears to brighten almost in phase. This suggests that blinkers are often 
(at least apparently) larger than a single CDS pixel. Therefore, we have grouped together neighbouring pixels showing almost simultaneous brightenings that satisfy the criteria for a blinker. "Almost simultaneous" means that they overlap in time by more than some minimum fraction of their individual lifetimes (we choose $80 \%$, see below). In the extreme case one pixel "blinks" completely inside the time range of the brightening of its neighbouring pixel. If this criterion is satisfied then the brightness events in the 2 neighbouring pixels are grouped together. This procedure is repeated with all other neighbouring pixels until no more pixels satisfying the criterion of simultaneity are found. We now define a blinker as a simultaneous brightening of such a group of pixels. Note that if more than one brightness event (separated in time) is identified for a given pixel then the search for simultaneously blinking neighbouring pixels is carried out for each such blinker separately.

The minimum percentage of overlap between neighbouring pixels at which they are still considered simultaneous is a free parameter which is determined as follows. We vary the minimum percentage of the overlap from $10 \%$ to $100 \%$ and calculate the number of independent blinkers with a size of 1 pixel and the total number of independent blinkers (i.e. groups of simultaneously blinking pixels including single pixels) found for each value. These numbers are relatively constant until a percentage of approximately $80 \%$. For higher minimum percentages these numbers increase rapidly, suggesting that such a requirement may be too stringent in view of the uncertainty in the starting and ending times introduced by noise.

Next we determine the spatially averaged light curve of each blinker. Using this light curve we then repeat steps $1-4$. In some cases (less than $10 \%$ of the total number) the spatially averaged light curve does not fulfill the blinker criteria, although the light curves of the individual pixels do. The main reason for this is that averaging can significantly smooth a light curve. In particular when the peak brightness in different pixels occurs at different times it can happen that the maximum brightness of the averaged light curve lies below the threshold. We suspect that in such cases our criterion for temporal overlap between the brightenings in different pixels is not stringent enough, so that two (or more) blinkers that happened close to each other in time and space have been mistakenly grouped together. Hence we regrouped the pixels in each such "nonblinker", but now using a changed definition of "simultaneous" brightening: the lifetimes of the blinkers in the two individual pixels overlap by at least $60 \%$ and one of them occurs completely within the lifetime of the other. This more stringent criterion successfully separated the previously overlapping blinkers in a reasonable manner in almost all these cases, as manual checks showed.

With Fig. 2 we illustrate some of the problems we encountered while identifying and grouping blinkers (as defined by our criteria) and show that some blinkers were time-structured. The meaning of the horizontal lines is the same as in Fig. 1. In Fig. 2 (left) portions of light curves are shown belonging to 4 different pixels whose addresses $(x, y)$ are given in brackets. The applied threshold is 1.45 and in the given time interval the code identified blinkers at the locations $(13,57),(13,58)$ and $(13,59)$. The pixel $(13,56)$ does not harbour a blinker at that time according to our criteria. The left and right vertical lines represent the start and end times, respectively, of the corresponding blinkers. All 3 start times are equal. The end times of pixels $(13,58)$ and $(13,59)$ are the same and similar to the end time of pixel $(13,57)$. The difference in these end times amounts to 1 time-step or $0.52 \mathrm{~min}$. We call such blinkers having only one significant peak simple blinkers.

In the four frames on the right of Fig. 2 we show an example of a complex blinker. Again, the threshold is 1.45 . The blinkers detected at locations $(2,124),(2$, $125),(2,126)$ and $(3,125)$ are part of a blinker region which comprises 7 pixels in all. The light curves of these blinkers are more complex than in the previous case, with 2 distinct peaks prior to $195 \mathrm{~min}$, and a third peak at later times, which however belongs to another blinker (according to our criteria). The start and end times, as well as the peaks, show some deviations from one pixel to another, but they fulfill the criteria described in this section to be grouped together. The deduced duration of the blinker at location $(2,126)$ is much shorter than the durations at the other three locations, but again, according to the chosen criteria this blinker is grouped with the others. We allow that it is arguable if such cases should belong to the same blinker or not, although the similarity between the 4 light curves confirms that it is reasonable to bunch them together. By visually inspecting complex blinkers we observed that their sizes are often larger than 15 pixels and that the significant sub-peaks are separated by between 5 and 10 min (cf. Fig. 1). In addition, the different subpeaks are also strongest at different spatial locations (e.g. Fig. 2). We expect that these are in fact multiple, partly overlapping events. If the spatial and temporal distribution of blinkers is relatively random, then it is likely that a certain number of such overlapping events are to be found in our sample. We further expect that due to the spatial smearing in CDS data, caused by the broad point-spreadfunction of CDS (Thompson 1998), the sizes of blinkers are enhanced and the number of overlapping blinkers is artificially increased. We have, however, found no satisfactory way of separating such overlapping blinkers from each other.

\subsection{Threshold determination}

We now describe how the threshold was selected. The chosen threshold needs to simultaneously satisfy three different partly contradictory criteria: a) A dominant fraction of the blinkers needs to be significant at the $3 \sigma$ level, i.e. the ratio $R=\left(I_{\max }-I_{\text {avg }}\right) / \sigma>3$, where $I_{\max }$ is the maximum brightness. This is best fulfilled for high thresholds. b) The total number of significant peaks (before spatial grouping), $N_{\text {tot }}$, should be as large as possible in order to 

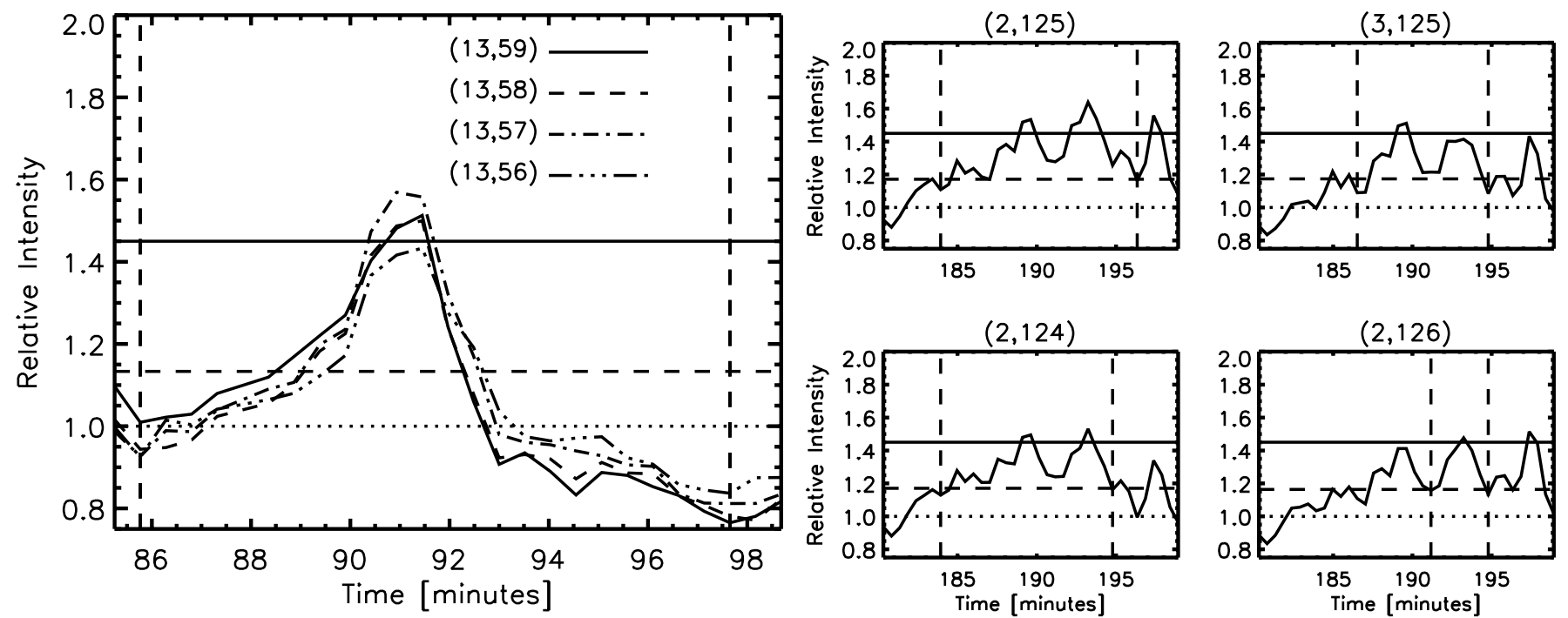

Fig. 2. Examples of light curves of simple (left) and complex (four smaller frames on the right) blinkers. See the caption of Fig. 1 for the meaning of the vertical and horizontal lines. Light curves refer to the locations (pixel numbers) given in the brackets $(x, y)$.

have better statistics. c) $N_{\text {tot }}$ and the "blinker filling factor" should be similar for the three spectral lines, so that the comparison between lines is easier. The blinker filling factor is the fraction of the total observed area within which a blinker is taking place at a given time. After applying a large set of thresholds to the three spectral lines, we found that the combination of these conditions is best fulfilled for the following thresholds: 1.3 for He I, 1.6 for $\mathrm{O} \mathrm{V}$ and 1.15 for $\mathrm{Mg}$ IX. The different levels of the thresholds in the three lines are already a sign of the different levels of brightening in them (compare, e.g., with Brković et al. 2000).

In the first part of this paper we only discuss the results of an analysis of $\mathrm{O} \mathrm{V}$. For this discussion we can neglect the third condition and find that a threshold of 1.45 is more appropriate. It maximizes the number of blinkers, while maintaining that over $90 \%$ of the blinkers have a signal to noise $>3$.

Of course, a certain measure of subjectivity remains when setting these thresholds. Choosing other thresholds mainly affects the number of blinkers and the average values of some of their parameters, but leaves the main results unchanged.

\subsection{Blinker parameters}

Next we proceed with the inspection of the temporal and spatial characteristics of blinkers and the determination of the parameters characterising them: the average brightness, energy, spatial size, duration and ratio of maximum to average brightness. These parameters are determined from the average light curves defined in Sect. 3.2, except for the size.

Note that the intensity ratio (and hence the energy) of a blinker depends on the chosen threshold: the number of blinkers changes with the threshold, and hence also the background brightness and, consequently, the start and end times. E.g., for the $\mathrm{O} \mathrm{V}$ line the maximum ratio $\left(I_{\max }\right)$ for a threshold of 1.45 was 4.51 , while for a threshold of 1.6 it was 4.78 .

While estimating the blinker energy our primary interest was the evaluation of the power law indices of energy distributions, explained in the Sect. 4.5, and not the estimate of absolute thermal energies. We determine the energy radiated in a particular line and suppose that the total energy released in a blinker is proportional to it. Because we cannot calculate the complete energy released by a blinker this assumption, which must not be true, was necessary. An alternative for the evaluation of the blinker energy is to follow the method of Krucker \& Benz (1998) which gives an estimate of the thermal energy. We find their method not to be applicable in our case because our and their definition of a brightening is quite different.

The energy radiated in a particular blinker is estimated as follows: we integrate the intensity times $\cos \theta(\theta$ is the heliocentric angle, i.e. the angle between the line of sight to the observer and the normal to the solar surface) over the upper hemisphere to obtain the flux in units of $\mathrm{erg} / \mathrm{cm}^{2} / \mathrm{s}$. Following the tradition used in astrophysics for the flux we give the result as the product of $\pi$ and the part independent of the integration boundaries. By the flux of a blinker we mean the excess flux, i.e. we calculate the flux for each pixel relative to the background flux. This flux is integrated over time as indicated by the shaded areas in Fig. 1, to give the integrated flux in units of $\mathrm{erg} / \mathrm{cm}^{2}$. Note that when the light curve dips below the background level the area bounded by background and light curve is subtracted (negative excess flux or flux deficit; black regions in the figure). The energy of a blinker is the sum over the integrated fluxes of all the pixels deemed to belong to this particular blinker so that the energy is finally given in units of erg. The solar surface area corresponding 

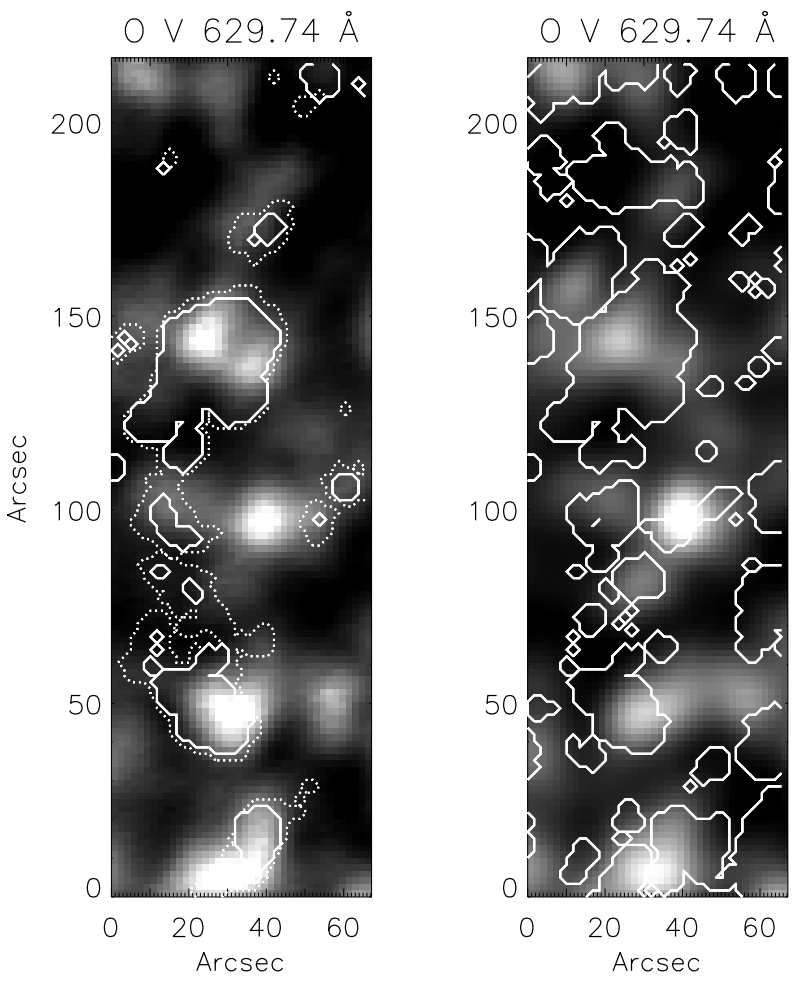

Fig. 3. Sample frame (left) and time-average (right) of a quietSun CDS movie in O V $629 \AA$ recorded on 3rd December 1996. The contours on the left frame depict the blinkers present at the time step of greatest blinker activity (corresponding to the selected frame; 204 minutes after beginning the observations), while the contours on the right frame depict all the blinkers present at some time during the 4 hours of the observations. Solid contours are obtained when a threshold of 1.6 is applied, dotted contours for a threshold of 1.45. Brighter areas correspond to higher intensities.

to 1 square pixel is $\approx 1.4 \times 10^{16} \mathrm{~cm}^{2}$ since $1^{\prime \prime}=718 \mathrm{~km}$ for the two dates of our observations.

\section{Results: Properties of blinkers seen in $\mathrm{O} \mathrm{V}$}

We begin by presenting some general properties of blinkers such as their spatial and temporal characteristics. For this we used the $\mathrm{O} \mathrm{V}$ line since blinkers are most prominent in radiation from the transition region. In addition to the threshold of 1.6 (see Sect. 3.3) we have also analysed blinkers with maximum brightness above a threshold of 1.45 in the second part of this section. This is the optimum threshold if we drop condition c) in Sect. 3.3. After the analysis of blinkers seen in $\mathrm{O} V$ we inspect, in Sect. 5.1, the behaviour of the He I and Mg IX lines in the space-time coordinates of the O V blinkers. Finally, we also consider brightenings in the $\mathrm{He} \mathrm{I}$ and $\mathrm{Mg}$ IX lines that lie above the thresholds of 1.3 and 1.15 , respectively, and then analyse the properties of these "blinkers", including the co-spatial, co-temporal behaviour of $\mathrm{O} \mathrm{V}$.
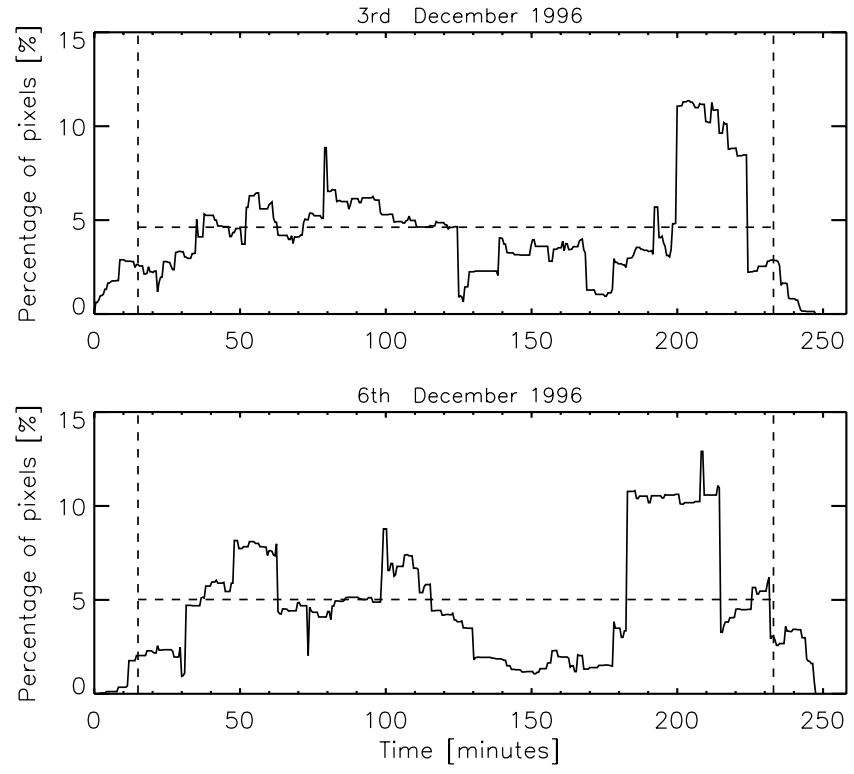

Fig. 4. Percentage of pixels showing $\mathrm{O} \mathrm{V}$ blinker activity in the field of view as a function of time. The results are reliable only between the vertical dashed lines. The horizontal dashed lines represent the average values of the data between the two vertical dashed lines, $4.6 \%$ and $5.0 \%$ for the upper and lower panel, respectively.

\subsection{Spatial locations}

The left image of Fig. 3 shows a sample frame of a quietSun CDS movie in O V $629 \AA$ at the moment when the number of points harbouring blinkers is maximum for a threshold value of 1.6. The solid contours outline the points which harbour blinkers at that time. We have also outlined the locations of blinkers when the threshold of 1.45 was applied (dotted contours). As expected, these contours enclose the solid contours ${ }^{1}$. In the right image of Fig. 3 the time-average of the quiet-Sun CDS movie is plotted. The contours enclose all the points which brighten sufficiently to be classified as blinkers at some time during the 4 hours of the observations (threshold of 1.6). We see that blinkers occur in bright as well as in dark regions, i.e., in the network and in the intranetwork, respectively.

\subsection{Temporal distributions}

In Fig. 4 we plot the fraction of pixels in the field of view showing blinkers as a function of time when a threshold of 1.6 is applied. Since some blinkers started before the beginning or ended after the end of our observations their starting and ending times cannot be determined and consequently they are not recorded as blinkers. A striking example is the bright blob in the middle of the right frame of Fig. 3. Note that on the left-hand image it is less bright. This very prominent brightening happened very close to the end of our time series. Therefore, the percentage of

\footnotetext{
${ }^{1}$ Exceptions may arise when blinkers are rejected because their beginning or end times lie outside the observed time interval. These times depend on the threshold; see Sect. 3.4.
} 

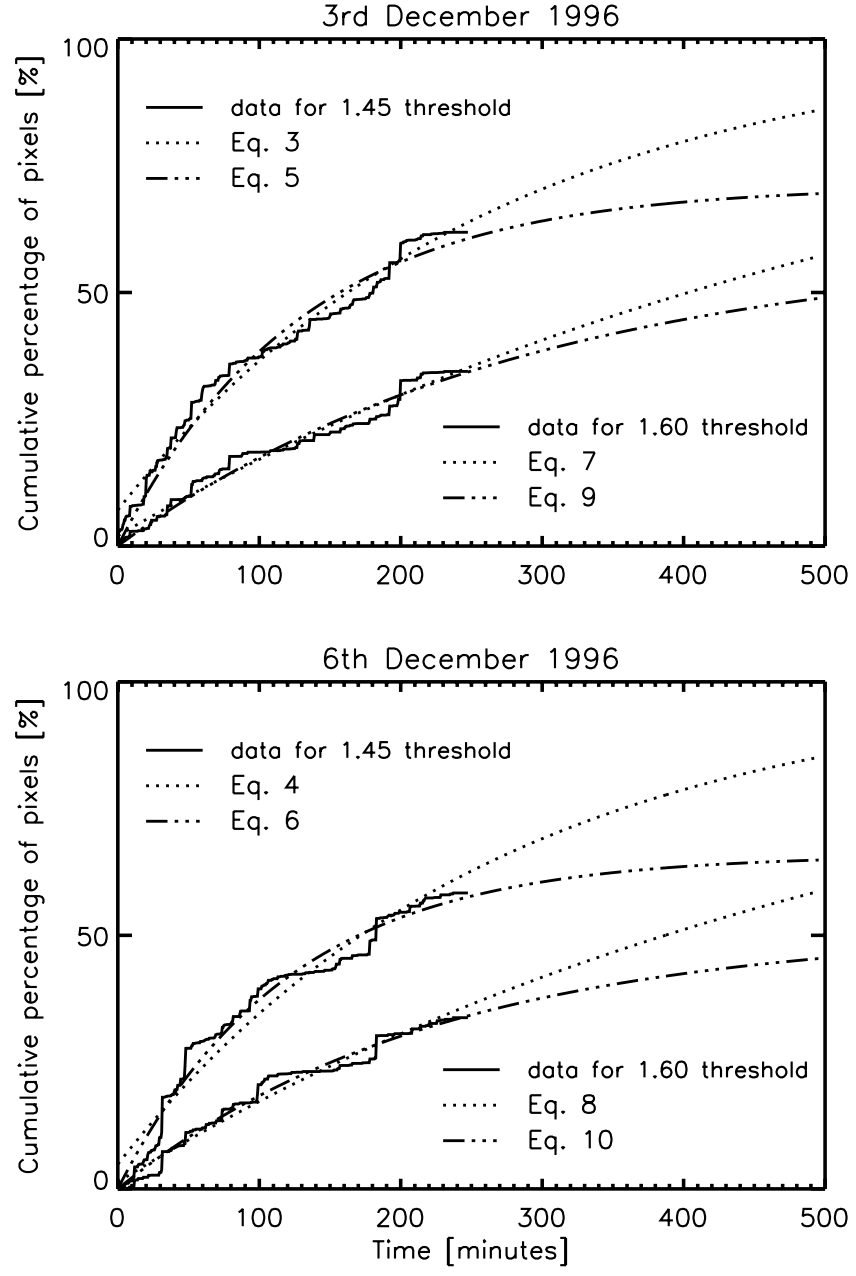

Fig. 5. Cumulative percentage of pixels harbouring blinker in the field of view as a function of time. The two frames refer to the two different days on which data were recorded. The upper curves in each plot are obtained with a threshold of 1.45 , the lower curves with a threshold of 1.6 .

pixels associated with blinkers is only reliable between the vertical dashed lines of Fig. 4. The percentage of pixels harbouring blinkers shows large temporal variations as expected if blinker activity takes place randomly. For the left frame of Fig. 3 this percentage amounts to $11.4 \%$ and on average approximately $5 \%$ of pixels harbour blinkers at any given time. For comparison, contours on the right frame of Fig. 3 cover $34 \%$ of the total area. There was always a blinker present in the field of view in the time interval between the vertical lines in Fig. 4. This is in agreement with Harrison et al. (1999) who found that before a blinker at one site is finished it is likely that another blinker occurs at another location in a sufficiently large observed area.

\subsection{Spatial and temporal distribution}

Figure 5 shows the cumulative fraction of pixels in the field of view showing blinkers as a function of time when thresholds of 1.45 and 1.6 are applied. Solid lines represent the data. The question that one hopes to answer from such a plot is whether, given a sufficient amount of time, eventually every point on the (quiet) solar surface exhibits a blinker or not. On a time scale much longer than the lifetime of supergranules (i.e. $\gg 20 \mathrm{~h}$ ) or than the time scale on which quiet-Sun magnetic flux is replaced $(\gg 40 \mathrm{~h}$, Schrijver et al. 1997) we expect this condition to be fulfilled in any case, but it is unclear whether the solar surface is filled with blinkers (at the CDS spatial resolution!) on time-scales shorter than the supergranule lifetime. Note that this question differs from that asked by Brković et al. (2000) and Rabin \& Dowdy (1992), who were interested in the surface fraction showing "significant" variability, respectively brightening, whereby the term "significant" depends on the details of the observations, in particular the noise level. Here we check which surface fraction is covered by brightenings above a fixed threshold.

In an attempt to find an answer we use two different functions to fit the data. One is of the form

$f_{\mathrm{c}}=100-a \cdot \mathrm{e}^{-t / t_{0}}$,

the other is of the form

$f_{\mathrm{c}}=a\left(1-\mathrm{e}^{-t / t_{0}}\right)$.

Here $f_{\mathrm{c}}$ is the cumulative fractional area exhibiting a blinker (in \%), $a$ and $t_{0}$ are free parameters, the latter describing a time scale. According to the first fit we expect each point in the field of view to blink after some time, while the second has a saturation level smaller than $100 \%$ if $a<100$. The two fits are represented by the dotted and dot-dashed lines in Fig. 5, respectively. The obtained numerical values of the free parameters are:

for a threshold of 1.45

3 Dec. : $f_{c}=100-92.9 \cdot \mathrm{e}^{-t / 262}$,

6 Dec.: $f_{\mathrm{c}}=100-95.1 \cdot \mathrm{e}^{-t / 267}$,

3 Dec. : $f_{\mathrm{c}}=71.0\left(1-\mathrm{e}^{-t / 129}\right)$,

6 Dec. $: f_{\mathrm{c}}=65.9\left(1-\mathrm{e}^{-t / 120}\right)$,

for a threshold of 1.6 :

3 Dec. : $f_{c}=100-98.0 \cdot \mathrm{e}^{-t / 600}$,

6 Dec.: $f_{\mathrm{c}}=100-98.7 \cdot \mathrm{e}^{-t / 570}$,

3 Dec. : $f_{\mathrm{c}}=59.5\left(1-\mathrm{e}^{-t / 288}\right)$,

6 Dec. : $f_{\mathrm{c}}=51.0\left(1-\mathrm{e}^{-t / 224}\right)$.

Equations (3), (4), (7) and (8) are fits of the type of Eq. (1) while Eqs. (5), (6), (9) and (10) are fits corresponding to Eq. (2). Both fits are very similar to each other for the period of the observations. They only diverge significantly if we extrapolate the fits to times well beyond the end of the observed time series. Nevertheless, with the present data we find that at least $2 / 3$ of the solar area harbours a blinker (at a threshold of 1.45) within a period of time 

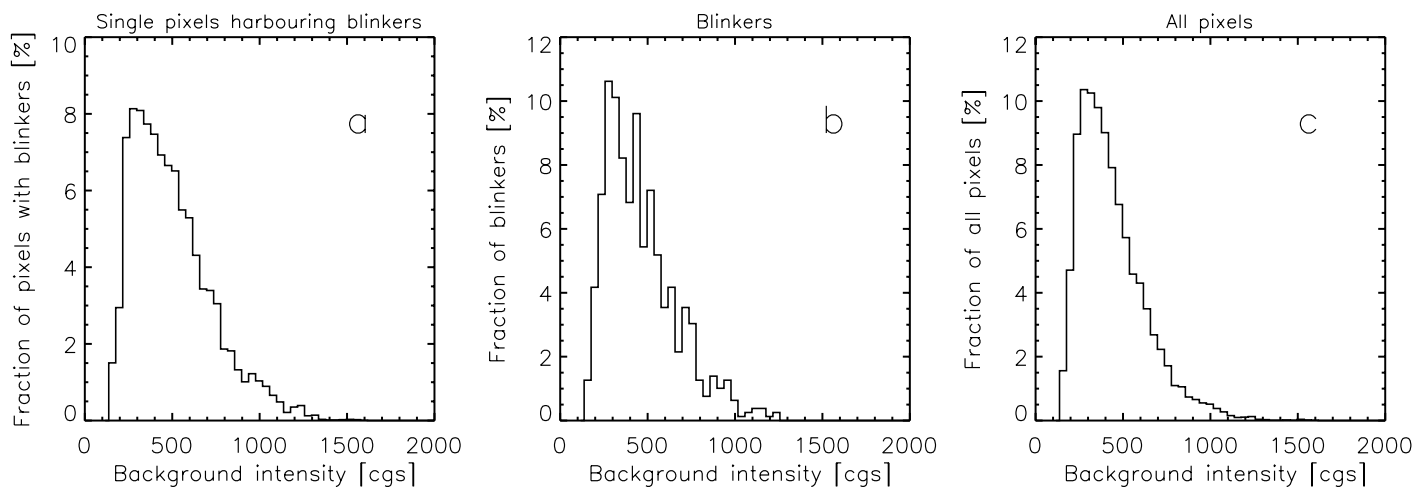

Fig. 6. Background intensity distributions for single pixels harbouring blinkers a), for blinkers b) and for all pixels c). The intensity is given in cgs units, i.e. in $\mathrm{erg} / \mathrm{cm}^{2} / \mathrm{sr} / \mathrm{s}$.
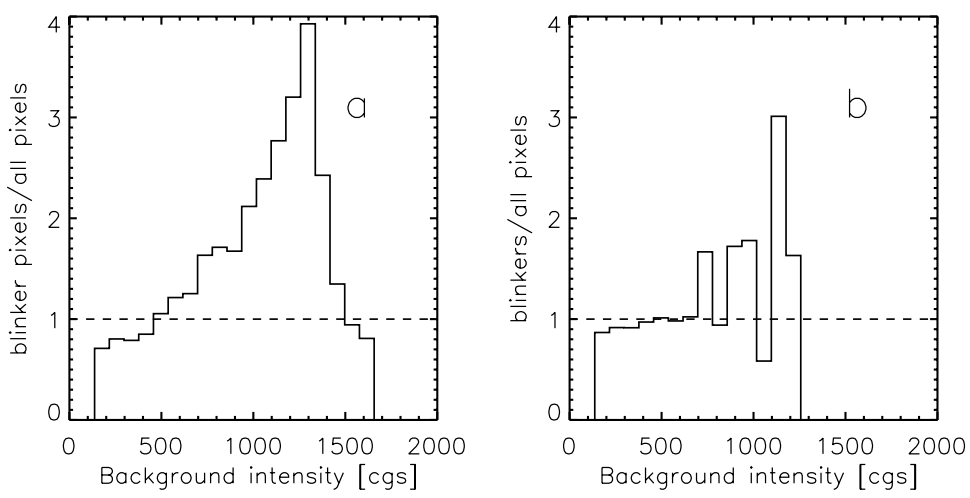

Fig. 7. Histograms of the number of blinkers at a given background intensity, normalized to the number of pixels having the same background intensity. The intensity is given in $\mathrm{cgs}$ units, i.e. in $\mathrm{erg} / \mathrm{cm}^{2} / \mathrm{sr} / \mathrm{s}$. We distinguish between the number of pixels harbouring blinkers a) and blinkers, i.e. conglomerates of simultaneously brightening pixels b).

less than a supergranule lifetime. The data sets from the 2 days give consistent results. Interestingly, increasing the threshold not only increases the time it takes for a given $f_{\mathrm{c}}$ to be reached, but, if the equation allows it, also lowers the asymptotically reached $f_{\mathrm{c}}(t=\infty)$. Only longer time series of images will provide more accurate results.

\subsection{Blinker parameters and their correlations}

In the remainder of this section we restrict ourselves to presenting results obtained from blinkers in the $\mathrm{O} \mathrm{V}$ data obtained using a threshold of 1.45. Our statistical sample is composed of 790 blinkers for the two data sets. For each blinker we determined the background intensity $I_{\mathrm{b}}$, its energy $E$, spatial area $A$, ratio of maximum to background brightness $R$ and duration $T$ as described in Sect. 3.4. Also, we determined the background intensity of each pixel irrespective of whether it harbours a blinker or not in the interval of the observations.

Some blinkers were located at the edge of the field of view and their size and energy could not be determined. The final results are affected insignificantly, i.e. within the uncertainty, by whether these blinkers were included in the analysis or not. We present the results including these blinkers.
Figure 6 shows histograms of background intensities for a) single pixels harbouring a blinker, b) groups of pixels forming a blinker (represented by the spatially averaged light curve) and c) all pixels. Any difference in shape of either of the first two and the third distribution is an indication of whether blinkers are preferably located in darker or brighter parts of the quiet Sun. An inspection of the figure shows that such differences are probably present, but are rather subtle. Therefore we consider ratios of histograms. In Fig. 7a we plot the ratio of the histograms shown in Figs. 6a and c, in Fig. 7b the ratio of the histograms in Figs. $6 \mathrm{~b}$ and $\mathrm{c}$ as a function of the background intensity. Figure 7 suggests that although blinkers are found in both network and intranetwork, there is a tendency for them to be concentrated in the brighter regions, whereby the trend is more clearly visible in the single pixels, possibly due to the better statistics. Figure 7a also suggests that the number of blinkers drops again in the very brightest regions, although the statistics become quite poor there. How this dependence on background brightness can be brought into agreement with the fact that the relative brightness variability is independent of brightness (Brković et al. 2000) still needs to be worked out.

Next we consider the correlations between the parameters characterising blinkers. The times and locations of blinkers were determined after applying a threshold of 1.45 
to the $\mathrm{O} \mathrm{V}$ line. These times and locations were then used to determine the values of corresponding parameters in He I and Mg IX. Table 1 gives the correlation coefficients. Only 3 quantities, namely $E, R$ and $A$ are significantly correlated (i.e. correlation coefficient $>0.5$ ) to each other, whereby the correlation between $E$ and $A$ is largest, while $I_{\mathrm{b}}$ and $T$ appear to be unrelated to each other and to the other parameters.

Thus, although blinkers are more common in brighter areas they are otherwise independent of background intensity.

The extremely high correlation between blinker energy and area is intriguing. Unless it is (partly) due to instrumental effects it implies that the energy of a blinker is determined primarily by the volume of the gas which brightens and only to a smaller extent by the amount by which a parcel of gas brightens (indicated by the correlation between $E$ and $R$ ).

Table 1. Cross-correlation coefficients for different pairs of parameters for the 3 spectral lines.

\begin{tabular}{|c|c|c|c|}
\hline parameters & He I & O V & Mg IX \\
\hline$E-T$ & 0.16 & 0.17 & 0.14 \\
\hline$E-I_{\mathrm{b}}$ & 0.05 & 0.08 & 0.03 \\
\hline$E-R$ & 0.51 & 0.73 & 0.47 \\
\hline$E-A$ & 0.88 & 0.92 & 0.79 \\
\hline$T-A$ & - & 0.15 & - \\
\hline$I_{\mathrm{b}}-R$ & -0.09 & 0.05 & -0.02 \\
\hline$I_{\mathrm{b}}-A$ & 0.06 & 0.07 & 0.03 \\
\hline$I_{\mathrm{b}}-T$ & -0.21 & -0.23 & -0.06 \\
\hline$R-T$ & 0.35 & 0.31 & 0.21 \\
\hline$R-A$ & 0.56 & 0.81 & 0.54 \\
\hline
\end{tabular}

\subsection{Frequency distributions}

We consider now the frequency distributions of the parameters characterising blinkers and represent most of them by power laws. Power law fits have been used in several studies (e.g. Drake 1971; Crosby et al. 1993 and references therein) to represent frequency of occurence distributions of different flare parameters, such as peak flux, integrated flux, rise time and decay time.

Parnell \& Jupp (2000) have described the problems met when studying frequency distributions of brightenings. In all frequency distribution studies one of the main problems is the choice of bin size, since it influences the final distribution. In some cases the way how some parameter is measured helps in the choice of the bin size. This is the case when a parameter can achieve only discrete values, e.g., sizes of blinkers are integer numbers (of pixels) while their durations are integer multiples of the sampling time, i.e. half a minute for our observations.

In contrast, the choice of bin sizes for the ratio of intensity to background and energy distributions is not so straightforward since the parameters achieve continuous values. We impose an upper limit on the bin size by requiring that no bin contains more than $50 \%$ of the points and that there are at least 20 bins. The thus obtained upper limits are $\pi \cdot 2.63 \times 10^{21} \mathrm{erg}$ and 0.075 for energy and ratio of intensity to background distributions, respectively. To obtain a lower limit we decreased the bin sizes in steps of $\pi \cdot 7.32 \times 10^{19} \mathrm{erg}$ and 0.0025 , respectively. For each bin size we fitted the data with power law functions and checked the quality of the fits, as represented by the $\chi^{2}$. Below bin sizes of $\pi \cdot 1.17 \times 10^{21} \mathrm{erg}$ and 0.040 for energy and ratio of intensity to background distributions, respectively, the quality of the fits distinctly decreased. These values are taken as lower limits for the bin sizes.

The frequency distributions of $E, A$ and $R$ are plotted in Fig. 8 based on all the analysed data. Each of these distributions can be well represented by a power law, i.e.

$\Delta P=C X^{\alpha} \Delta X$,

where $X$ is $E, R$ or $A, \alpha$ is $\alpha_{E}, \alpha_{R}$ or $\alpha_{A}$ and $\Delta P=$ $\Delta N * 100 / N$. Here $\Delta N$ is the number of points in a given bin, $X+\Delta X$, and $N$ is the total number of points, so that $\Delta P$ gives the fraction of points in that bin. The fits in the $\log -\log$ plots are given by the dashed lines. Plotted in Fig. 8d is the duration distribution. It is better represented by a log-normal distribution than a power-law (details are given below). All these values are, of course, dependent on the employed definition of blinkers.

For the distribution of the energy we studied the distributions obtained for different bin sizes in the range $\pi\left(1.17 \times 10^{21}, 2.63 \times 10^{21}\right) \mathrm{erg}$. The corresponding values of the slope $\alpha_{E}$ lie in the range $(-1.23,-1.51)$. In general a larger bin size gives a smaller $\alpha_{E}$. Hence, the slope changes by $23 \%$, while the bin size changes by $250 \%$. Figure $8 \mathrm{a}$ shows a plot in which a bin size of $\pi \cdot 1.67 \times 10^{21} \mathrm{erg}$ is used. The corresponding slope $\alpha_{E}=-1.34$. The average energy of blinkers amounts to $\pi \cdot 3.66 \times 10^{22} \mathrm{erg}$. It should be noted that the spatial overlap between what are probably independent brightenings, which causes them to be counted to the same blinker (see Sect. 3.2), probably enhances the number of more energetic blinkers leading to an underestimate of the deduced slope.

The ratios of intensity to background lie in the range $1.45-4.53$, with an average value 1.65 . The minimum and maximum ratio as well as the average are influenced by the threshold. Harrison et al. (1999) found an average ratio of 1.48 for the same line. Considering that we calculate the ratio differently from Harrison et al. (1999) and also set the threshold in a different way, the two values are reasonably similar. For the determination of power law fits we applied different bin sizes in the range $(0.040,0.075)$ and obtained values of $\alpha_{R}$ between -7.34 and -8.00 . Here we notice a $9 \%$ change in the slope for a $188 \%$ change in the bin size. The ratio of intensity to background distribution is by far the steepest of all the distributions. Thus, the number of blinkers decreases far more rapidly with increasing $R$ than with energy or size. A plot with a bin size of 0.05 is shown in Fig. 8b. The corresponding slope $\alpha_{R}=-7.82$. 

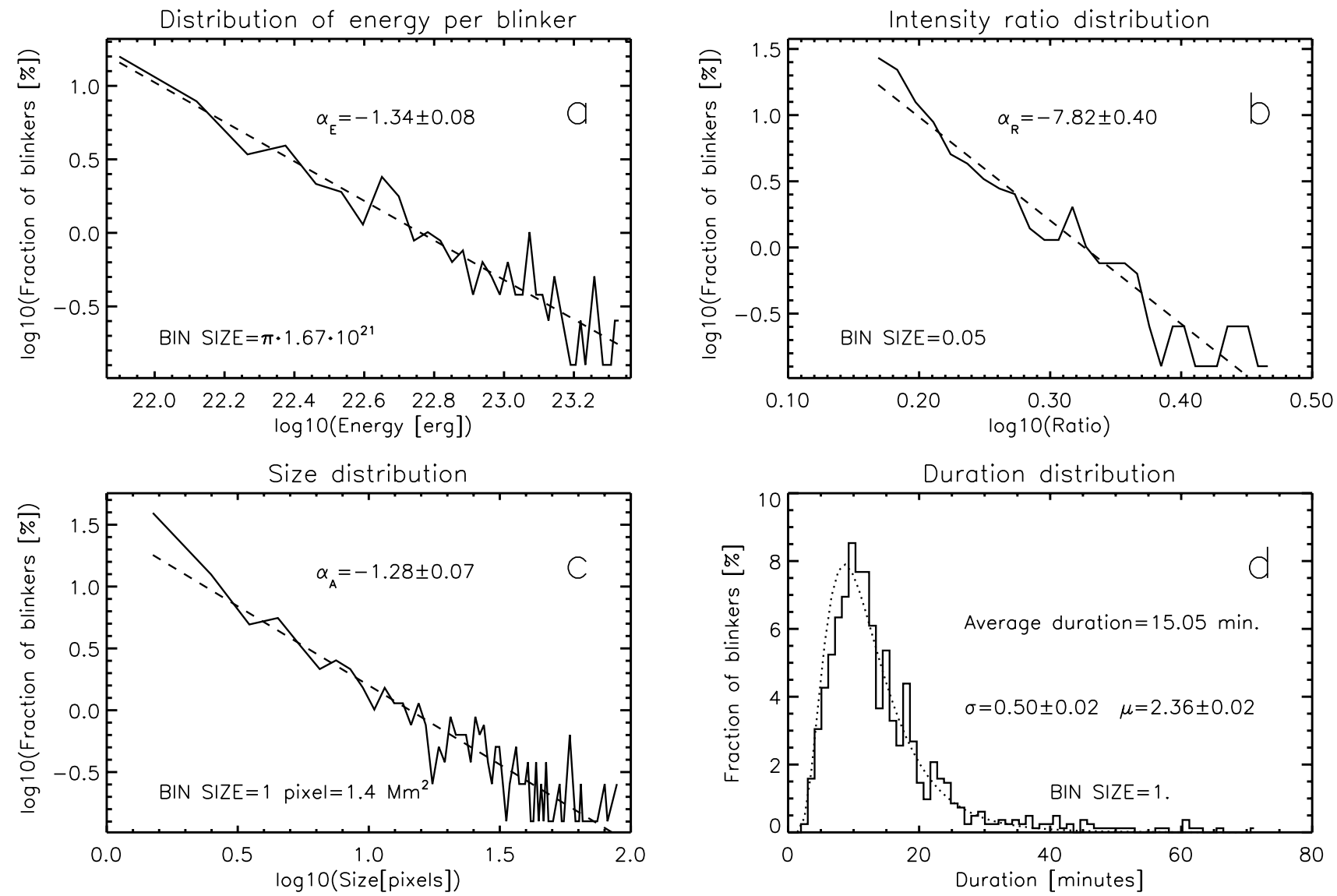

Fig. 8. $\log -\log$ plots of the frequency distributions of a) energy, b) size or area and c) ratio of intensity to background of the 790 observed blinkers. d) histogram of the duration. The solid curves in the log-log plots represent logarithms of percentages and the dashed lines are power law fits to the data points (see Eq. (11)). The dotted line in frame d is a log-normal fit to the data points (see Eq. (12)). The bin sizes, the values of the exponents of the power law fits, $\alpha$, and the values of the parameters $\sigma$ and $\mu$ of the log-normal fit are given in each frame.

The histogram of the size distribution is characterised by a peak at the size of 1 pixel $\left(1.4 \mathrm{Mm}^{2}\right)$, which harbours $40 \%$ of all blinkers, and an extended tail (see the log-log distribution in Fig 8c). The average value of the size was found to be 16.1 pixels or $22.3 \mathrm{Mm}^{2}$, but if we exclude the $5 \%$ largest blinkers from the analysis, we find that the average blinker size is reduced to 7.3 pixels. These $5 \%$ largest blinkers encompass over 60 pixels each and they more than double the average size. Both average values are smaller than the "typical" blinker size of $5 \times 5$ pixels given by Harrison et al. (1999).

The shortest and longest blinker lifetimes are 3 and 110 min, respectively. The shortest duration which can be detected in our data is approximately 1 min so that the lifetimes of all blinkers are well resolved. Obviously the minimum size of blinkers lies below the spatial resolution of CDS, but the temporal resolution of our observations appears to be sufficient to resolve almost all blinkers. The typical duration of a blinker lies between 5 and $20 \mathrm{~min}$, with an average value of $15.0 \mathrm{~min}$. Harrison et al. (1999) found that this average value is about $40 \mathrm{~min}$, while their most common blinkers last $17 \mathrm{~min}$. They got their typical blinker after exclusion of extended tails from the distributions of blinker parameters. Note, however, that the Harrison et al. study could not detect many of the shorter duration events. This may account for much of the difference between their results and ours.

We cannot completely rule out that the drop in number of events with short duration (Fig. 8d) may be an artifact of the selected threshold of 1.45 for the ratio of intensity to background, since there is a weak correlation (at the 0.3 level in the correlation coefficient) between $T$ and $R$. The lifetime distribution is well represented by a log-normal function,

$\Delta P=\frac{100}{\sqrt{\pi} \sigma T} \cdot \mathrm{e}^{-\frac{(\ln T-\mu)^{2}}{2 \sigma^{2}}} \cdot \Delta T$.

Here $\Delta P$ is the percentage of points with durations between $T$ and $T+\Delta T$. The values of the parameters $\sigma$ and $\mu$ are given in the figure. Log-normal distributions have been employed in solar physics by, e.g., Bogdan et al. (1988), Martínez Pillet et al. (1993) and Pauluhn et al. (2000). Of particular relevance here is the work of Martínez Pillet et al. (1993), who found that another temporal parameters, namely the decay rate of sunspots is log-normally distributed. 

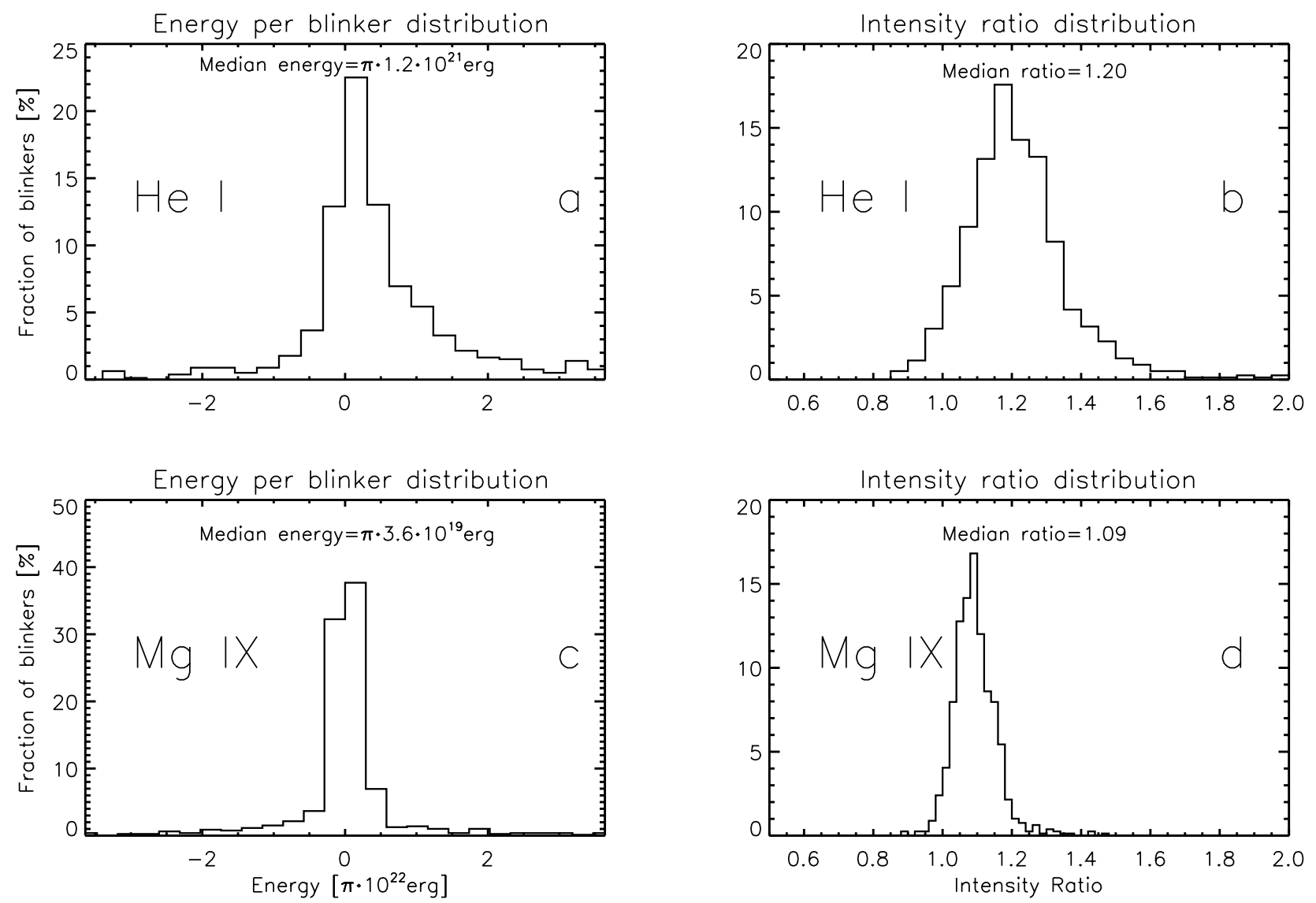

Fig. 9. Distributions of energy and ratio of intensity to background for the He I and $\mathrm{Mg}$ IX lines during O V blinkers; see the distributions in Fig. 8.

We now follow Harrison et al. (1999) and estimate the number of blinkers present on the solar surface at any given time. We detected 790 blinkers in a total of $29926 \mathrm{~s}$ within the field of view $\left(67^{\prime \prime} \times 217^{\prime \prime}\right)$, or approximately $3.5 \times 10^{-12}$ events $/ \mathrm{km}^{2} / \mathrm{s}$. The solar surface area is $6.3 \times 10^{12} \mathrm{~km}^{2}$. Thus, 22.1 events per second start over the entire solar surface. With an average duration of $15.0 \mathrm{~min}$ per blinker we can expect about 20000 blinkers, as defined by our criterion, to be present at any moment on the solar surface. The much smaller number of blinkers, namely 3000, obtained by Harrison et al. (1999) illustrates just how sensitive this number is to the criteria used for the identification of blinkers and for separating closely located blinkers from each other.

\section{Results: Comparison between spectral lines}

\subsection{He I and $\mathrm{Mg} I X$ signatures of $O \mathrm{~V}$ blinkers}

In this section we look for the signatures in the He I and $\mathrm{Mg}$ IX lines of the blinkers found in the $\mathrm{O} \mathrm{V}$ line using a threshold of 1.45. To this end we consider the pixels and time intervals associated with blinkers in the $\mathrm{O} \mathrm{V}$ line and determine the energy and intensity ratio in the He I and $\mathrm{Mg}$ IX line radiation, as explained in Sect. 3. Recall that the energy is calculated relative to the background intensity, which, also for He I and Mg IX, is averaged over all times at which there is no blinker in $\mathrm{O} \mathrm{V}$ (at least for the analysis presented in this section). Since it can happen that the brightness in He I or Mg IX is lower during an $\mathrm{O} \mathrm{V}$ blinker than at other times the excess energies in the chromospheric and coronal lines can be negative. Figure 9 shows histograms of energy and intensity ratio for the He I (Figs. 9a,b) and the Mg IX line (c,d). The distributions in both lines are almost symmetric, unlike the results found for the $\mathrm{O} \mathrm{V}$ line. Negative energies are present in $\mathrm{He} \mathrm{I}$ in $24 \%$ of the $\mathrm{O} \mathrm{V}$ blinkers and in $45 \%$ in $\mathrm{Mg}$ IX. Intensity ratios less than 1 are also observed in both lines and average values of the ratio amount to 1.22 for He I and 1.09 for Mg IX. In both cases we found higher values than Harrison et al. (1999), who published values of 1.08 and 1.04, respectively. Our ratio distributions are also broader than theirs.

The excess energy in none of the lines correlates to that in another line. The highest correlation of 0.26 is found between He I and Mg IX. On the other hand we notice a significant correlation between maximum intensity ratios, with correlation coefficients greater than 0.5 for each line pair. In Fig. 10 we plot the intensity ratio of one line against the intensity ratio of another. This figure reveals that those $\mathrm{O} \mathrm{V}$ blinkers for which the intensity ratio in the $\mathrm{He} \mathrm{I}$ and $\mathrm{Mg}$ IX lines is $\leq 1$ have intensity 

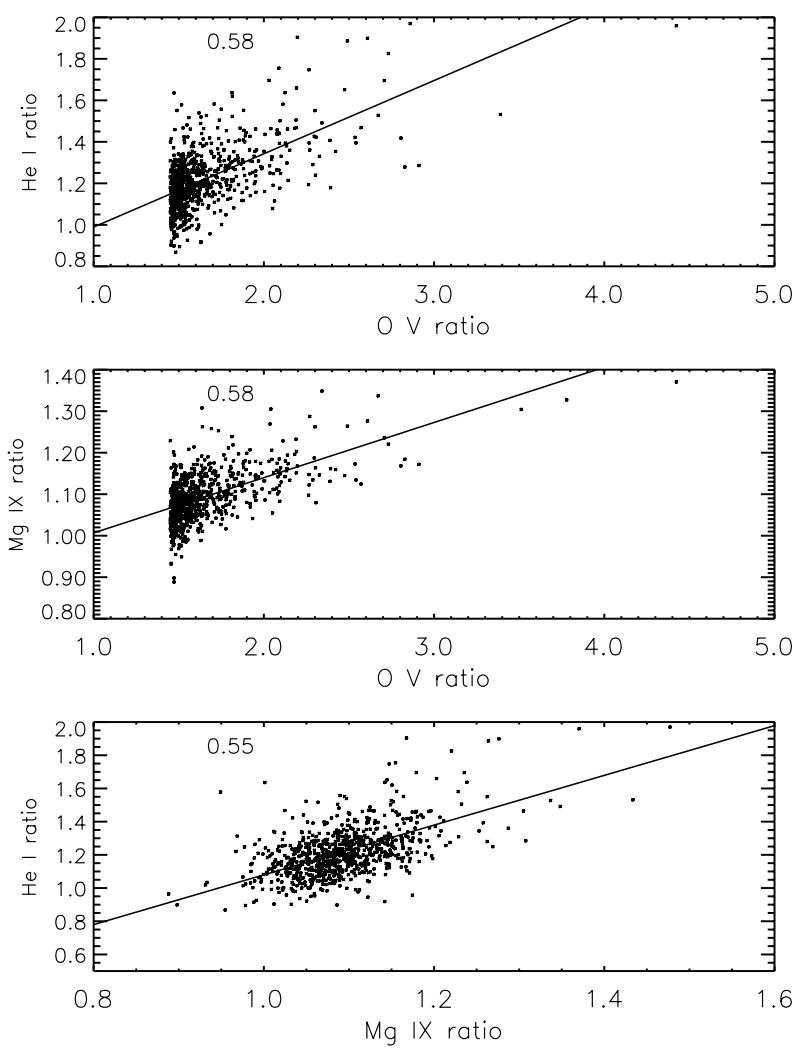

Fig. 10. Maximum intensity ratio during an $\mathrm{O} \mathrm{V}$ blinker in one spectral line vs. that in another line. The solid lines are linear least-squares fits to the data. Correlation coefficients are printed at the upper left corner of each frame.

ratios in $\mathrm{O} \mathrm{V}<1.8$. Note that for many of these events noise may be responsible for the $R<1$ values in He I and $\mathrm{Mg}$ IX. However, it appears unlikely that noise is entirely, or even dominantly responsible for the negative energies in $\mathrm{He} \mathrm{I}$ or $\mathrm{Mg}$ IX associated with many $\mathrm{O} \mathrm{V}$ blinkers. The large number of such events and the general lack of a correlation suggests that $\mathrm{O} \mathrm{V}$ blinkers are not simply a transition-region response to micro-flares or other brightenings taking place in the corona, or to the deposition of energy flux in the chromosphere, but are to a certain extent an independent phenomenon. The correlation seen in the intensity ratio implies, however, that the brightenings at the 3 temperatures are not completely independent. To make this conclusion more certain we still need to check how brightenings seen in Mg IX and/or He I are visible in $\mathrm{O}$ V. Such an analysis will be presented in Sect. 5.2.

We have also investigated the correlations between the various parameters of He I alone and Mg IX alone. The cross-correlation coefficients are given in Table 1 along with those of $\mathrm{O} \mathrm{V}$. The only exception is the correlation between $A$ and $T$, since these quantities are both determined directly from $\mathrm{O} \mathrm{V}$ data. The $\mathrm{He} \mathrm{I}$ and $\mathrm{Mg}$ IX correlation coefficients mirror the behaviour seen for $\mathrm{O} \mathrm{V}$, although the correlations are somewhat poorer.

\subsection{Brightenings observed in the $\mathrm{He} / 584 \AA$ and $\mathrm{Mg} I X 368$ A lines: How do they relate to O V 629 Å blinkers?}

After looking for the signature produced by $\mathrm{O} \mathrm{V}$ blinkers in He I and Mg IX in Sect. 5.1 we now carry out the complementary analysis and look for blinkers (or similar brightenings) directly in the $\mathrm{He} \mathrm{I}$ and $\mathrm{Mg}$ IX data sets. Thresholds are chosen according to the three criteria given in Sect. 3: we employ 1.3 for He I and 1.15 for Mg IX. Then the identical procedure used to find $\mathrm{O} \mathrm{V}$ blinkers is also applied to the data in these lines. For purposes of comparison we here repeat the analysis of the $\mathrm{O} \mathrm{V}$ data set, but for a threshold of 1.6, which gives a similar number of pixels harbouring blinkers as in the other two lines. The total number of blinkers observed in these lines for both data sets are 450, 300 and 1971 for He I, O V and Mg IX, respectively. Already the difference in these numbers suggests that brightenings in Mg IX are coherent over smaller spatial areas, or last for a shorter time. We now present the main results of an analysis that is similar to the one described in Sect. 4.

First we test whether the trend that blinkers are more common in brighter areas, deduced from the $\mathrm{O} \mathrm{V}$ data is also exhibited by the other lines (see Fig. 11). This trend remains clear in $\mathrm{O} \mathrm{V}$ if the threshold is increased to 1.6, but is at best marginal in He I and Mg IX brightenings.

In Fig. 12 we plot the frequency distributions of the parameters characterising the blinkers detected in the 3 lines. As in Fig. 8 a log-log representation is chosen, except for the duration. Qualitatively, Fig. 12 looks similar to Fig. 8, although the scatter is larger for the He I and $\mathrm{O} \mathrm{V}$ lines due to the smaller number of blinkers. The range covered by blinker energies is large in all the 3 lines although this is not visible from Fig. 12. The blinkers with highest energies were excluded from the evaluation of the energy fits (due to the poor statistics) and accordingly not plotted in the figure. The approximate ratios of maximum to minimum energy amount to 6200, 48000 and 43000 for He I, O V and Mg IX blinkers, respectively. The size distributions exhibit a prominent peak at one pixel, which contains $80 \%$ of blinkers in the case of Mg IX, and an extended tail. The largest blinker (lying outside the frame) is about 450 times larger than the smallest blinkers of size 1 square pixel. The average size of Mg IX blinkers is only 2 pixels. Either the brightenings seen in this line are on a much smaller spatial scale than those seen in the 2 other lines and are below the spatial resolution, or, due to the small contrast in this line the spatial smearing due to the broad point-spread-function of CDS (Thompson 1998) is less severe in this line.

The 1971 brightenings seen in Mg IX greatly outnumber those in He I (450) and in O V (300). They are distinctly smaller in amplitude and size, and of somewhat shorter duration. In contrast, the $\mathrm{He} \mathrm{I}$ and $\mathrm{O} \mathrm{V}$ blinkers exhibit similar properties, although the intensity ratio and size of the He I blinkers is somewhat smaller. The average values of blinker durations in all lines are smaller 
than the 40 min found by Harrison et al. (1999) and they decrease from the chromospheric $(23.2 \mathrm{~min})$ through the transition region (16.7 $\mathrm{min}$ ) to the coronal line (12.2 $\mathrm{min})$. Recall that the difference to Harrison et al. may arise from the fact that they could not detect many of the shorter duration events.
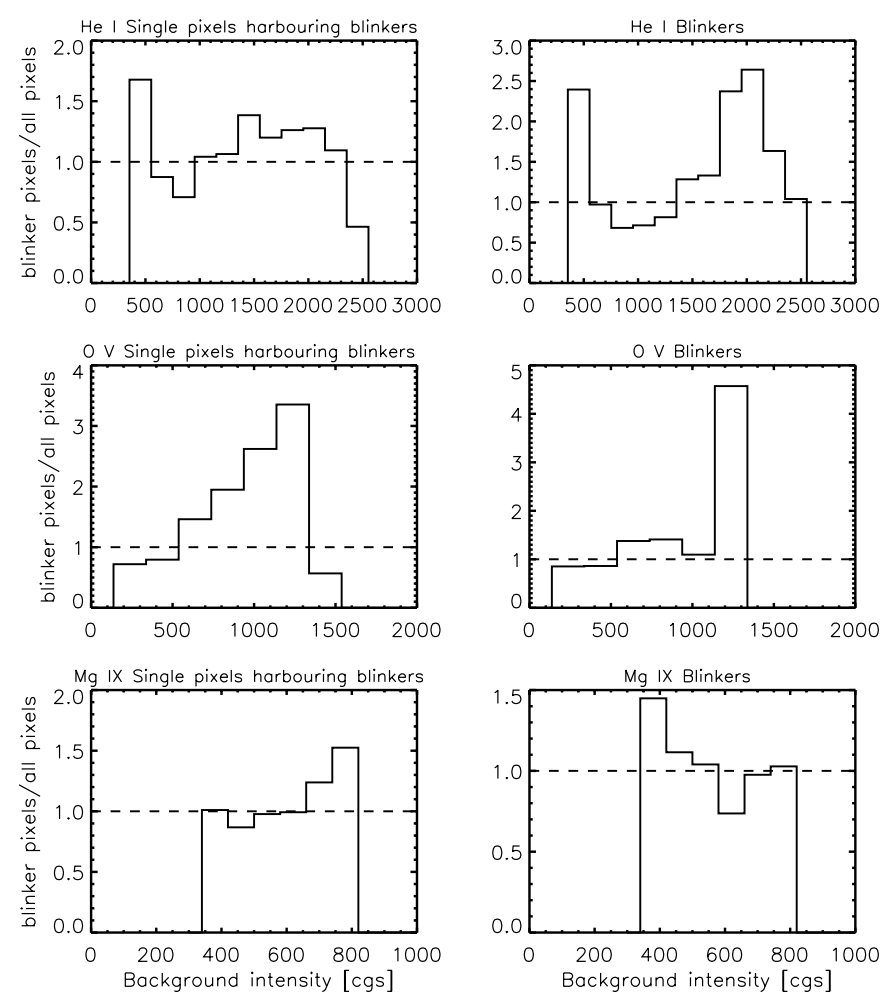

Fig. 11. Same as for Fig. 7, but now for He I (top), O V (with a threshold of 1.6; middle) and Mg IX (bottom).

The power law fits to the relevant distributions have been calculated in the same manner as described in Sect. 4 (see Eq. (11)). The parameter $\alpha$ (power-law exponent) and the applied bin size are given in each frame of Fig. 12. Following Sect. 4.5 we again tested the influence of the bin size on $\alpha$ for the distributions of energy and ratio. The results of these tests are given in Tables 2 and 3. There we tabulate the range in bin size, the corresponding range in $\alpha$, as well as $\delta \alpha(\%)$, the percentage change in $\alpha$ due to varying the bin size, and $\delta \alpha / \delta$ (bin size), a measure of the sensitivity of $\alpha$ on the bin size. The $\alpha_{E}$ for He I and $\mathrm{O} \mathrm{V}$ lie between -1.5 and -1 , while for $\mathrm{Mg}$ IX $\alpha_{E}<$ -2 appears most likely. In general Mg IX distributions follow the steepest power laws, while at the same time having exponents that exhibit the smallest dependence on bin size, while the $\alpha$ values obtained from $\mathrm{O} \mathrm{V}$ data are the most sensitive. In He I and $\mathrm{O}$ V the slopes for the energy and size power law fits are remarkably close to each other. We cannot estimate if the different power law indices found for He I, O V and Mg IX are real or an artifact due to an incomplete knowledge of the complete energy in a blinker. Improved estimates of this quantity would be very desirable.
The blinker duration distribution of Mg IX line data is satisfactorily represented by a log-normal function as in Sect. 4.5. The O V (threshold 1.6) and He I duration distributions are only poorly represented by such a function. Bearing in mind that the Mg IX line has by far the largest statistics (i.e. number of blinkers), we believe that it is the poor statistics which are responsible for the poor correspondence for the other two lines. This is supported by the fact that for $\mathrm{O} \mathrm{V}$ the improvement in the statistics obtained by lowering the threshold to 1.45 leads to a clear log-normal distribution (Sect. 4.5).

The correlations between the parameters describing blinkers deduced in the section are similar to the ones found for $\mathrm{O} \mathrm{V}$ when the threshold of 1.45 was applied (Table 1). Thus, the correlation coefficients between the pairs involving the energy, ratio and size are very high, while the coefficients of correlations involving background intensity and duration are small.

Table 2. Influence of bin size on $\alpha_{E}$, the power law exponent of the frequency distribution of blinker energy.

\begin{tabular}{lccc}
\hline Line & He I & O V & Mg IX \\
\hline bin sizes $\left(\pi \cdot 10^{20}\right)$ & $12-29$ & $18-52$ & $2.2-4.4$ \\
\hline values $\alpha_{E}$ & -1.15 to $-1.36-1.00$ to $-1.44-1.98$ to -2.24 \\
\hline$\delta \alpha_{E}(\%)$ & 18 & 44 & 13 \\
\hline$\delta \alpha_{E} / \delta($ bin size $)\left(10^{-23}\right)$ & 3.4 & 4.1 & 19 \\
\hline
\end{tabular}

Table 3. Influence of bin size on $\alpha_{R}$, the power law exponent of the frequency distribution of intensity ratios.

\begin{tabular}{lccc}
\hline Line & He I & O V & Mg IX \\
\hline bin sizes $\left(10^{-3}\right)$ & $30-50$ & $25-55$ & $9-14.5$ \\
\hline values $\alpha_{R}$ & -7.91 to -8.76 & -4.79 to -5.73 & -26.77 to -29.36 \\
\hline$\delta \alpha_{R}(\%)$ & 11 & 20 & 10 \\
\hline$\delta \alpha_{R} / \delta($ bin size $)$ & 5.5 & 6.7 & 18.2 \\
\hline
\end{tabular}

Next we investigate the spatial correlation between blinkers seen in different spectral lines. To this end we determine locations of blinking pixels for each line at each time step and find the common locations for each pair of lines. Next we divide the number of common locations by the total number of blinking pixels of each member of the line-pair. We thus obtain two ratios of which we take the average. This gives us the fraction of all blinkers that are simultaneous and co-spatial in two lines. He I and $\mathrm{O} \mathrm{V}$ exhibit the highest fraction of common blinkers, although even in this case less than $40 \%$ of the blinking pixels identified in one line are also seen in the other line. The average overlap for the He I and $\mathrm{Mg}$ IX and the $\mathrm{O} \mathrm{V}$ and $\mathrm{Mg}$ IX pairs is less than $25 \%$ and $20 \%$, respectively. Note that $\mathrm{Mg}$ IX is slightly better correlated with He I than with O V. For the given field of view totalling 5160 pixels and blinker filling factor of $5 \%$ we find that the probability that the fraction of common blinkers of $40 \%$ is entirely due to chance (assuming blinkers in both lines to be randomly 

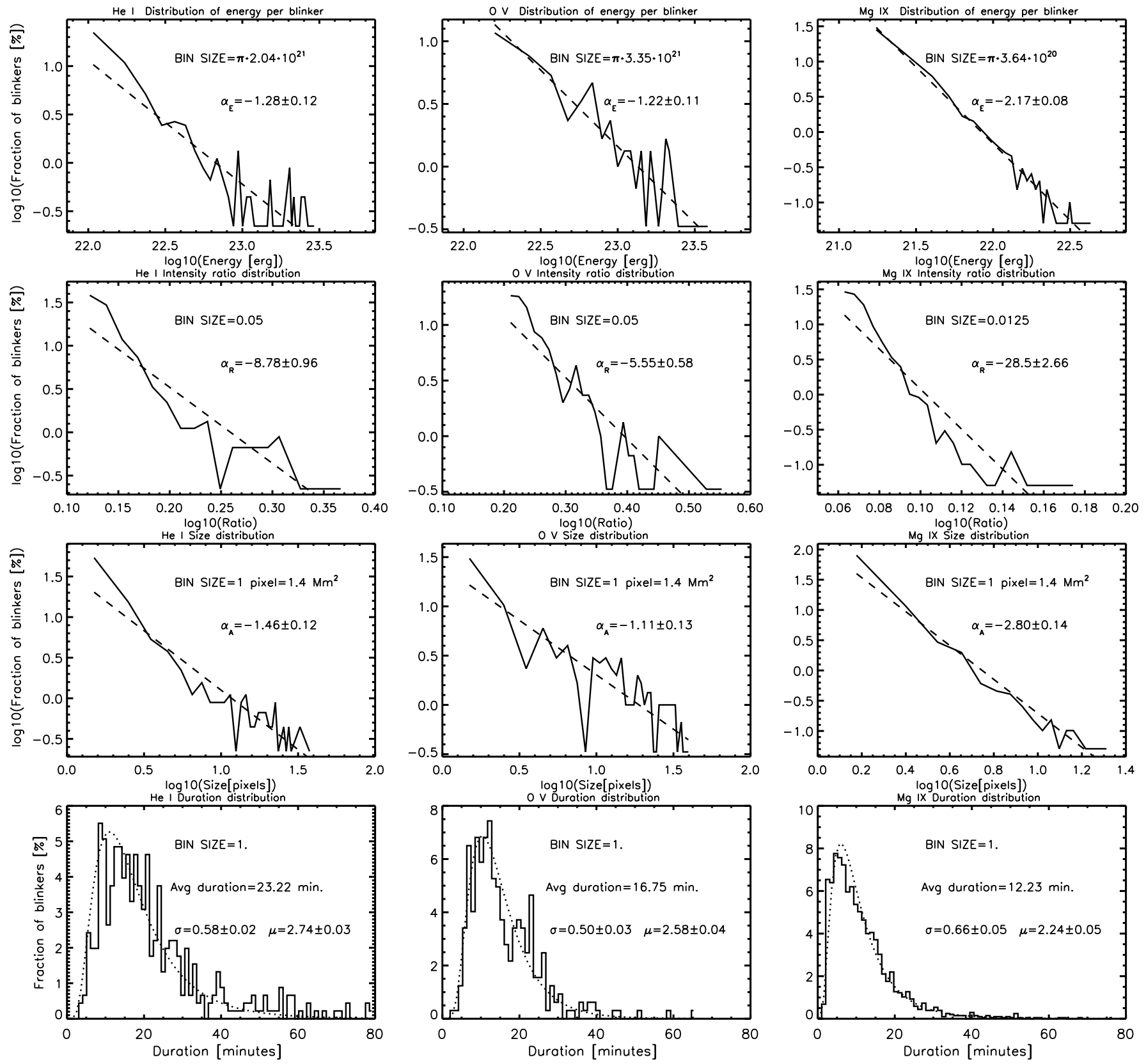

Fig. 12. Log- $\log$ plots of the frequency distributions of energy, size and intensity ratio, as well as histograms of the duration of the blinkers identified in He I, O V and Mg IX, respectively. Dashed lines are power-law fits to the data in the upper three rows of frames (see Eq. (11)). The dotted curves are log-normal fits to the duration distributions (see Eq. (12)). The values of the exponents of the power law fits, $\alpha$, as well as the values of the $\sigma$ and $\mu$ parameters of the log-normal fits and the bin sizes are indicated.

distributed) is less than $10^{-15}$. A similar low probability is obtained also for a lower fractional overlap of $20 \%$. This means that the spatial overlap between blinkers in different spectral lines, even if it is relatively small, is real and is not a purely random effect.

From the number of detected blinkers in He I, O V and Mg IX, the length of the time series and the field of view we get that $12.7,8.4$ and 55.5 events per second start over the entire solar surface as seen in the He I, $\mathrm{O} \mathrm{V}$ and $\mathrm{Mg}$ IX lines, respectively. From the corresponding average durations of blinkers, (cf. Fig. 12), the above numbers transform to about 17500 (He I), $8500(\mathrm{O} \mathrm{V})$ and 40500 (Mg IX) events present at any moment in time over the entire solar surface. If we compare the $8500 \mathrm{O} \mathrm{V}$ blinkers, found with a threshold of 1.6 (above) with the 20000 blinkers obtained for a threshold of 1.45 (Sect. 4.4), the sensitivity of the number of blinkers to the threshold is reinforced. This dependence is even more striking in the case of Mg IX. E.g., if the threshold is increased to 1.2 the number of expected events decreases to 7300 , a reduction by a factor of 5.6. Recall that the signal to noise ratio of the Mg IX line data is the smallest of the three lines. 


\section{Discussion: Blinkers as compared with other EUV transient brightenings}

Here we consider other kinds of transient activity and relate them to the blinkers studied here. We first compare our results with those of Harrison et al. (1999). They recorded 97 blinkers in $190000 \mathrm{~s}$ within two fields of view of $40^{\prime \prime} \times 100^{\prime \prime}$ and $40^{\prime \prime} \times 124^{\prime \prime}$, respectively, which they scanned with a $4^{\prime \prime} \times 240^{\prime \prime}$ slit. Both investigations show that blinkers are most prominent in transition region lines, particularly in $\mathrm{O} \mathrm{V}$, in which enhancements in intensity reach a factor of 5 . In particular, brightenings in He I and $\mathrm{Mg}$ IX are not as strong as in $\mathrm{O} \mathrm{V}$. Average durations of a blinker are on the order of tens of minutes in both reports (40 min according to Harrison et al., about 16 min here). The size of their "typical" blinker is $5 \times 5$ pixels, while the average area covered by our $\mathrm{O} \mathrm{V}$ blinkers is about 16 pixels. The rate at which blinkers occur on the solar surface is smaller according to Harrison et al. $\left(1.24 \mathrm{~s}^{-1}\right.$ over the entire solar surface compared to $9 \mathrm{~s}^{-1}$ and $22 \mathrm{~s}^{-1}$ for thresholds of 1.6 and 1.45 , respectively). A possible reason for these different rates lies in the different methods used for blinker identification. Harrison et al. define the pixel groups of interest visually and then search for a blinker signature when the $\mathrm{O} \mathrm{V}$ intensity of the pixel or the pixel group rises at least $10 \%$ above the average of the last three measurements. The end time of a blinker is defined in a similar way: when the intensity drops to within $10 \%$ of the pre-event intensity. Our work compares intensity enhancements to the average intensity taken over all time steps without blinker activity. The minimum amounts of enhancements in our $\mathrm{O} \mathrm{V}$ cases are $45 \%$ and $60 \%$. Our automated procedure has the advantage that it allows us to detect blinkers in the network and the intranetwork, while Harrison et al. find blinker sites only in the network. For $\mathrm{O} \mathrm{V}$ we find that blinkers are more common in the network, but certainly not restricted to there.

Berghmans et al. (1998) also report on transient brightenings. They used two wavelength bands of the Extreme-Ultraviolet Imaging Telescope (EIT), the $304 \AA$ band with the He II line $(80000 \mathrm{~K})$ and the $195 \AA$ band with the Fe XII line $\left(1.6 \times 10^{6} \mathrm{~K}\right)$. The pixel size was $2.6^{\prime \prime} \times 2.6^{\prime \prime}$ and the observed field of view $14^{\prime} \times 14^{\prime}$. Hence they consider one temperature which lies between those covered by He I and $\mathrm{O} \mathrm{V}$ in our data, and one truly coronal temperature lying outside the range considered here. The average sampling time was something more than a minute. The observations in the 2 lines were not simultaneous. The analysed data sets consisted of 173 images in He II and 55 in Fe XII. These images were first submitted to an one-dimensional discrete Fourier transform along the time dimension and later to an automated brightening detection scheme. In its basics their detection scheme is similar to ours. For each brightening they determined the duration, spatial area, peak intensity and radiative loss. The upper limit for the duration of brightenings was $60 \mathrm{~min}$ in their He II sequence and $30 \mathrm{~min}$ in the Fe XII sequence. The lower limit on duration was $2 \mathrm{~min}$ in both sequences, set by the sampling. The frequency distributions of all determined parameters (except the peak intensity) followed power laws. For the duration distributions they applied a bin size of $132 \mathrm{~s}$ and found power-law exponents -3.1 (He II) and -2.1 (Fe XII). These results differ qualitatively from ours, since we find a log-normal representation for blinker durations. While analysing spatial areas of the brightenings they applied bin sizes of $5 \mathrm{Mm}^{2}$ and $14 \mathrm{Mm}^{2}$ for He II and Fe XII sequences, respectively. The corresponding power-law indices were -2.7 (He II) and -2.0 (Fe XII). The index for He II differs from those of the cooler lines reported in this work, -1.5 (He I), -1.1 and $-1.3(\mathrm{O} \mathrm{V})$. We obtain -2.8 for $\mathrm{Mg}$ IX, but it is unclear to what extent this can be compared to the Fe XII result. They calculated that the radiative losses of the brightenings in the He II sequence range from $10^{25}$ to $10^{27} \mathrm{erg}$ and from $10^{24}$ to $10^{26} \mathrm{erg}$ in the Fe XII sequence. The power-law indices were -1.9 for the transition region line and -1.35 for the coronal line. For our distributions of blinker energies these indices were -1.22 for the transition region line and -2.17 for the coronal line. The results of the two investigations differ not only in numerical value, but also in the character, i.e. from lower (transition region) to higher (corona) temperatures their indices increase (i.e. power laws become flatter), while our decrease. Certainly, a part of this difference arises from the different ways of calculating these parameters. For the He II sequence they found that the rate at which the brightenings they detected occur is $20-40 \mathrm{~s}^{-1}$ over the whole Sun. This rate is close to the rates for $\mathrm{O} V$ presented here $9 \mathrm{~s}^{-1}$ and $22 \mathrm{~s}^{-1}$ for the two considered thresholds. Bearing in mind all differences between both observations one cannot expect a one-to-one correspondence in the final results. Nevertheless, presuming that the He II brightenings are related to our blinkers, the sometimes qualitative differences between the results of the two investigations are surprising.

Krucker et al. (1997) used simultaneous Yohkoh Soft X-ray Telescope (SXT) and Very Large Array (VLA) radio observations of the quiet Sun and reported on brightenings they called network flares. They estimated that the typical thermal energy content of these events amounts to $10^{26} \mathrm{erg}$ per event and they occur at a rate of one event per $3 \mathrm{~s}$ on the total solar surface. The existence of network flares was confirmed by Krucker \& Benz (1998), who alternatingly used the $171 \AA$ and $195 \AA$ bands of EIT on SOHO at a cadence of about $2 \mathrm{~min}$ during an observing time of $42 \mathrm{~min}$. These bands include the Fe IX/X $\left(1.3 \times 10^{6} \mathrm{~K}\right)$ and Fe XII $\left(1.6 \times 10^{6} \mathrm{~K}\right)$ lines. The pixel size was $2.6^{\prime \prime} \times 2.6^{\prime \prime}$ and the observed field of view $7^{\prime} \times 7^{\prime}$. For each pixel in both bands they determined the emission measure and temperature and converted the enhancements in emission measure into thermal energy content. They found that the largest areas of these events reach $49 \mathrm{Mm}^{2}$ and that the thermal energy content ranges from $8 \times 10^{24}$ to $2.8 \times 10^{26} \mathrm{erg}$. The distributions of these energies follow power-laws with indices between -2.6 and -2.3 . These indices are compatible with our result for $\mathrm{Mg}$ IX. 
Berghmans et al. determined the occurrence rate of the coronal brightenings seen in Fe XII and found $1.2 \mathrm{~s}^{-1}$. They proposed that these brightenings are the low-energy counterparts of the network flares. We find that the brightenings which we detect using Mg IX have a relatively high birth-rate, $55 \mathrm{~s}^{-1}$ and $10 \mathrm{~s}^{-1}$ for thresholds 1.15 and 1.2 , respectively. This suggests that our data are more sensitive to such brightenings. There is not enough evidence to claim that the Mg IX activity is directly related to network flares, but at the same time we cannot reject this possibility.

Brueckner \& Bartoe (1983) and Dere et al. (1984) identified a class of transient events, so-called EUV explosive events, which leave a typical signature in solar transition region lines. The usual spatial scale of these relatively short living events (from 20 to $200 \mathrm{~s}$ ) is on the order of a few arcsec and kinetic energies involved are on the order of $10^{23}$ to $10^{25} \mathrm{erg}$. During the events average line of sight velocities amount to $150 \mathrm{~km} \mathrm{~s}^{-1}$. The results of Innes et al. (1997) suggested a magnetic reconnection origin for the observed bi-directional plasma jets. Explosive events and blinkers are best observed in transition region lines. Other connections between them have not been reported. For example, the average duration of explosive events $(\approx 1 \mathrm{~min})$ is much shorter than of blinkers $(\approx 20 \mathrm{~min})$. Harrison et al. (1999) speculated that explosive events could be part of a blinker, but further work is required to test this. The birth rate of explosive events is about $500 \mathrm{~s}^{-1}$ over the whole Sun. This is an order of magnitude larger than for blinkers. No link between explosive events and network flares has so far been established.

We now discuss frequency distributions of parameters related to energies. Of particular importance is the exponent $\alpha_{E}$, since if $\alpha_{E}<-2$ then small, unresolved transient brightenings, such as microflares and nanoflares, may contribute significantly to the heating of the solar corona (Hudson 1991). The main characteristic of most power-law index studies in active regions (Drake 1971, Crosby et al. 1993 and references therein) is that the slope $\alpha_{E}$ is in the range $(-2,-1)$. For example, peak count rates from active region flares gave the index $\alpha_{E} \approx-1.8$, Dennis (1985). Hudson et al. (1969) and Drake (1971) found that soft X-ray peak fluxes of active region flares have $\alpha_{E}=-1.84$ and -1.75 , respectively. Shimizu (1995) found $\alpha_{E}$ between -1.6 and -1.5 in distributions of flare thermal energy inputs larger than $10^{27} \mathrm{erg}$ as determined from soft X-ray brightenings in active regions.

On the other hand, analysing quiet Sun data from EIT on SOHO Krucker \& Benz (1998) found that distributions of events with energies in the range $8 \times 10^{24}$ to $2.8 \times 10^{26}$ erg have $\alpha_{E}$ between -2.6 and -2.3 . Using quiet Sun data taken by TRACE (Transition Region And Coronal Explorer, Strong et al. 1994) Parnell \& Jupp (2000) analysed event energies in the range $10^{23}-10^{26}$ erg and also found a value of $\alpha_{E}<-2$. The present analysis has revealed that for the energies in the Mg IX data $\alpha_{E}<-2$ is the most likely value. The different results obtained for active region flares and for quiet Sun brightenings may not only reflect differences between these types of events, but may partly stem from the fact that the parameters analysed are different. Recall that in all these studies the energy is calculated either from spectral analysis or a DEM approach, i.e. the studies do not give the total energy. We find parameters (units), such as energy in erg (Shimizu 1995), total energy in electrons in erg (Crosby et al. 1993), peak flux in $\mathrm{erg} / \mathrm{cm}^{2} / \mathrm{s}$ (Drake 1971), integrated flux in $\mathrm{erg} / \mathrm{cm}^{2}$ (Drake 1971) or simply count rate in counts/s (Dennis 1985). If there is a correlation between lifetime and, e.g. peak brightness and size then an integration in time will give different distributions and correspondingly, in principle, a different value of $\alpha_{E}$. These details certainly make a direct comparison of different results more difficult.

Here we have also determined exponents of the power law fits to the energy distributions returned by the transition-region and chromospheric lines. These values are of interest if the heating of the upper chromosphere (or lower transition region), as sampled by He I, and of the middle transition region sampled by $\mathrm{O} V$ is due to local heating events.

Note that the power laws derived for blinkers and presumably also for other brightenings are probably too flat due to the inability to distinguish between neighbouring brightenings as a result of the limited spatial resolution of the observations. Due to this groups of smaller and thus less energetic blinkers are misidentified as larger blinkers.

\section{Conclusions and summary}

We have analysed the blinkers observed in quiet Sun CDS movies obtained simultaneously in He I $584.3 \AA$, O V $629.7 \AA$ and Mg IX $368.1 \AA$. In general, the qualitative properties of blinkers are independent of the applied threshold, but quantitatively the parameters are, of course, influenced by it. We applied intensity-ratio thresholds of 1.45 and 1.6 for O V, 1.3 for He I and 1.15 for Mg IX. The maximum flux enhancements were almost 5 in O V, 2.4 in He I and 1.6 in Mg IX. We have determined various parameters describing the blinkers. The main results of the analysis are:

- Blinkers are best detected in O V. Blinkers are present in bright as well as in dark regions. The blinkers in $\mathrm{O} \mathrm{V}$ and to a lesser extent also in He I are relatively more frequent in brighter areas. However, these blinkers do not differ significantly in property from those in darker areas.

- Energy, size and intensity ratio correlate well with each other, while other pairs of parameters show little correlation (the intensity ratio is the ratio of peak to background intensity). The highest correlation is between blinker size and energy, suggesting that it is the volume of the brightening gas which primarily determines the energy of a blinker.

- The frequency distributions of energy, size and ratio can be parameterised by power law fits, whereas the 
distribution of blinker durations can be described by a log-normal function.

- There is little spatial and temporal correlation between brightenings in $\mathrm{Mg}$ IX and $\mathrm{O} \mathrm{V}$ (or Mg IX and $\mathrm{He} \mathrm{I}$ ), which leads us to suggest that the $\mathrm{Mg}$ IX line does not register blinkers, but brightenings that on the whole are independent. Nevertheless, the $20 \%$ overlap between the brightenings in the 2 lines is significant.

- The lack of correlation between brightenings in the $\mathrm{Mg}$ IX and other lines could mean that the corona is at least partly decoupled from the transition region and chromosphere, in contrast to the classical model of the transiton region (Gabriel 1976; Fontenla et al. 1993), but in support of the more complex model by Dowdy et al. (1986).

- The area covered by a single blinker ranges from 1 pixel to as large as 450 pixels. The average is highest for $\mathrm{O} \mathrm{V}$, about $23.5 \mathrm{Mm}^{2}$, in He I it is $12.4 \mathrm{Mm}^{2}$ and in $\mathrm{Mg}$ IX $2.8 \mathrm{Mm}^{2}$. Most of the blinkers are localised in a single pixel; about $35 \%$ in $\mathrm{O} \mathrm{V}$, more than $50 \%$ in $\mathrm{He} \mathrm{I}$ and more than $80 \%$ in $\mathrm{Mg}$ IX. We allow that this result, as well as the large size of big blinkers can be influenced by our criteria for grouping individual pixels to form blinker.

- The minimum lifetime which our blinker identification procedure could identify was $1 \mathrm{~min}$. The lifetimes of blinkers in all the 3 lines range from 3 to $110 \mathrm{~min}$. In contrast to the results obtained by Berghmans et al. from EIT data we find blinker durations to be lognormally distributed, suggesting that very short blinkers are indeed rare. The average durations are between $10 \mathrm{~min}$ and half an hour, i.e., $23 \mathrm{~min}$ in He I, about 16 min in $\mathrm{O} \mathrm{V}$ and $12 \mathrm{~min}$ in $\mathrm{Mg} \mathrm{IX}$.

- The birth-rate of blinkers is the most threshold sensitive parameter and is different for each line. For the applied thresholds it is on the order of 10-50 events per second over the entire solar surface.

Acknowledgements. We would like to thank R. Harrison for carefully reading and commenting on the manuscript, and J. O. Stenflo and M. C. E. Huber for their encouragement and support. We are also grateful to the CDS team, as well as the SOHO command staff whose help was invaluable for obtaining these observations. SOHO is a mission of international cooperation between ESA and NASA. This work was partly supported by the Swiss National Science Foundation, grant No. 21-45083.95, and by a grant from the ETH-Zürich which is gratefully acknowledged.

\section{References}

Berghmans, D., Clette, F., \& Moses, D. 1998, A\&A, 336, 1039 Bogdan, T. J., Gilman, P. A., Lerche, I., \& Howard, R. 1988, ApJ, 327, 451

Brković, A., Rüedi, I., \& Solanki, S. K. 1999, in Proc. Eigth SOHO Workshop, Plasma Dynamics and Diagnostics in the Solar Transition Region and Corona, ed. J.-C. Vial, \& B. Kaldeich-Schürmann, ESA SP-446, 191

Brković, A., Rüedi, I., Solanki, S. K., et al. 2000, A\&A, 353, 1083

Brueckner, G. E., \& Bartoe, J.-D. F. 1983, ApJ, 272, 329

Crosby, N. B., Aschwanden, M. J., \& Dennis, B. R. 1993, Sol. Phys., 143, 275

Dennis, B. R. 1985, Sol. Phys., 100, 465

Dere, K. P., Bartoe, J.-D. F., \& Brueckner, G. E. 1984, ApJ, 281,870

Dowdy, J. F. Jr., Rabin, D., \& Moore, R. L. 1986, Sol. Phys., 105,35

Drake, J. F. 1971, Sol. Phys., 16, 152

Fontenla, J. M., Avrett, E. H., \& Loeser, R. 1993, ApJ, 406, 319

Gabriel, A. H. 1976, Phil. Trans. Roy. Soc. London A, 281, 339

Harrison, R. A., Sawyer, E. C., Carter, M. K., et al. 1995, Sol. Phys., 162, 233

Harrison, R. A. 1997, Sol. Phys., 175, 467

Harrison, R. A., Lang, J., Brooks, D. H., \& Innes, D. E. 1999, A\&A, 351, 1115

Hudson, H. S., Peterson, L. E., \& Schwartz, D. A. 1969, ApJ, 157,389

Hudson, H. S. 1991, Sol. Phys., 133, 357

Innes, D. E., Inhester, B., Axford, W. I., \& Wilhelm, K. 1997, Nature, 386, 811

Krucker, S., Benz, A. O., Bastian, T. S., \& Acton, L. W. 1997, ApJ, 488, 499

Krucker, S., \& Benz, A. O. 1998, ApJ, 501, L213

Martínez Pillet, V., Moreno-Insertis, F., \& Vázquez, M. 1993, A\&A, 274, 521

Parnell, C. E., \& Jupp, P. E. 2000, ApJ, 529, 554

Pauluhn, A., Solanki, S. K., Rüedi, I., Landi, E., \& Schühle, U. 2000, A\&A, 362, 737

Rabin, D., \& Dowdy, J. F. Jr. 1992, ApJ, 398, 665

Schrijver, C. J., Title, A. M., van Ballegooijen, A. A., et al. 1997, ApJ, 487, 424

Shimizu, T. 1995, PASJ, 47, 251

Strong, K., Bruner, M., Tarbell, T., Title, A., \& Wolfson, C. J. 1994, Space Sci. Rev., 70, 119

Thompson, W. 1998, CDS Software Note \# 49, Version 4, http://orpheus. nascom. nasa. gov/cds/sof tware_notes. html 